\title{
Heavy Quark Production at HERA
}
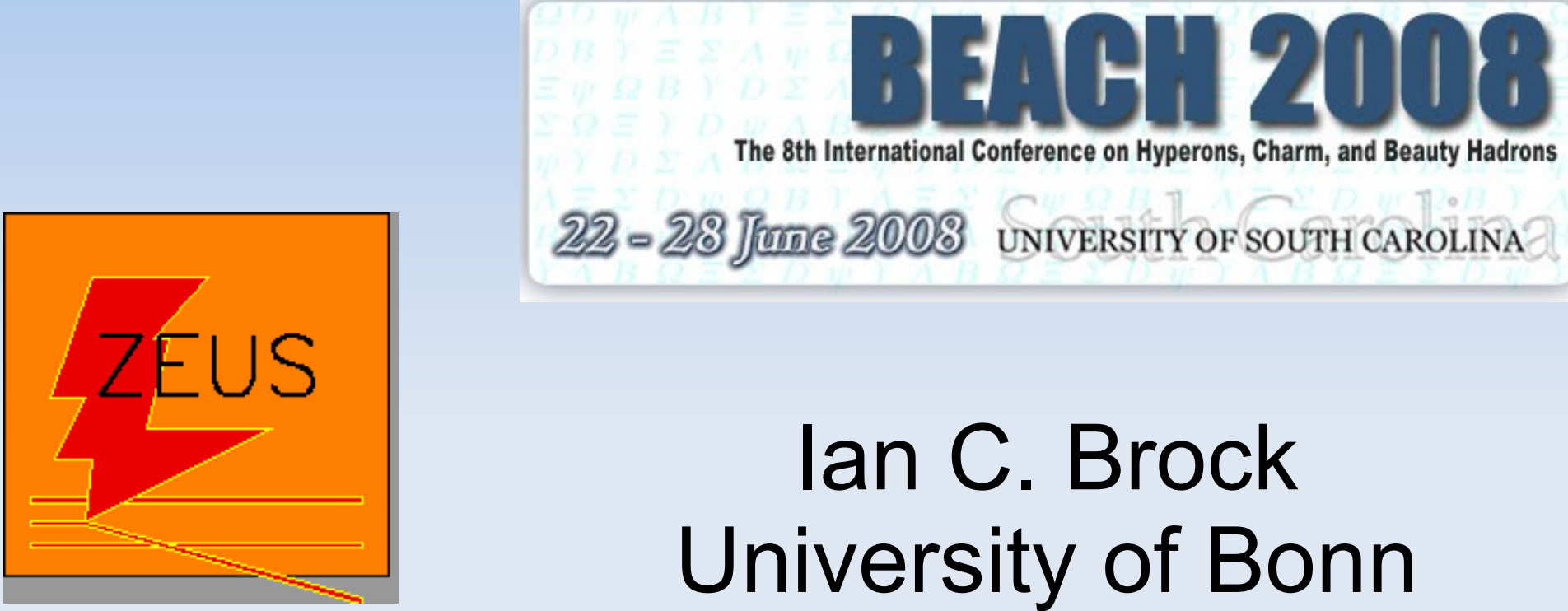

\section{Ian C. Brock University of Bonn}

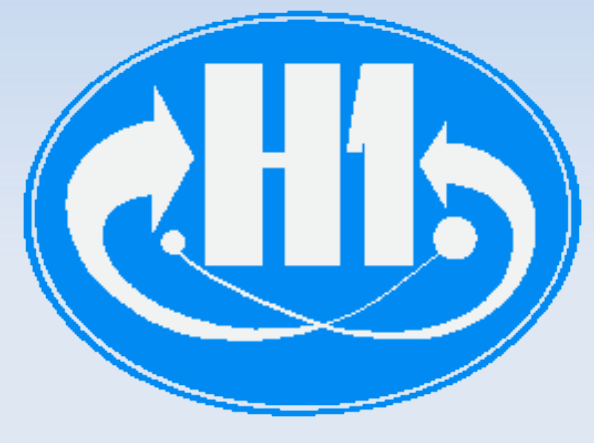

On behalf of the $\mathrm{H} 1$ and ZEUS Collaborations

\section{$24^{\text {th }}$ June 2008}

GEFÖRDERT VOM

武武

Bundesministerium für Bildung und Forschung 


\section{Outline}

- Introduction

- Charm via D*

- Beauty via semileptonic decays to $\mu$

- Charm and Beauty via semileptonic decays to e

- Beauty correlations

- $\mathrm{F}_{2}^{\mathrm{bb}}, \mathrm{F}_{2}^{\mathrm{cc}}$

- Conclusions \& Outlook 


\section{Heavy Flavour Production at HERA}

- Boson-gluon fusion (BGF) is main production mechanism

- Concentrate on studies of production mechanism:

- Test QCD (different hard scales, $\left.m_{\mathrm{Q}}, \mathrm{p}_{\mathrm{T}}, \mathrm{Q}^{2}\right)$

- Gluon Parton Density Function?

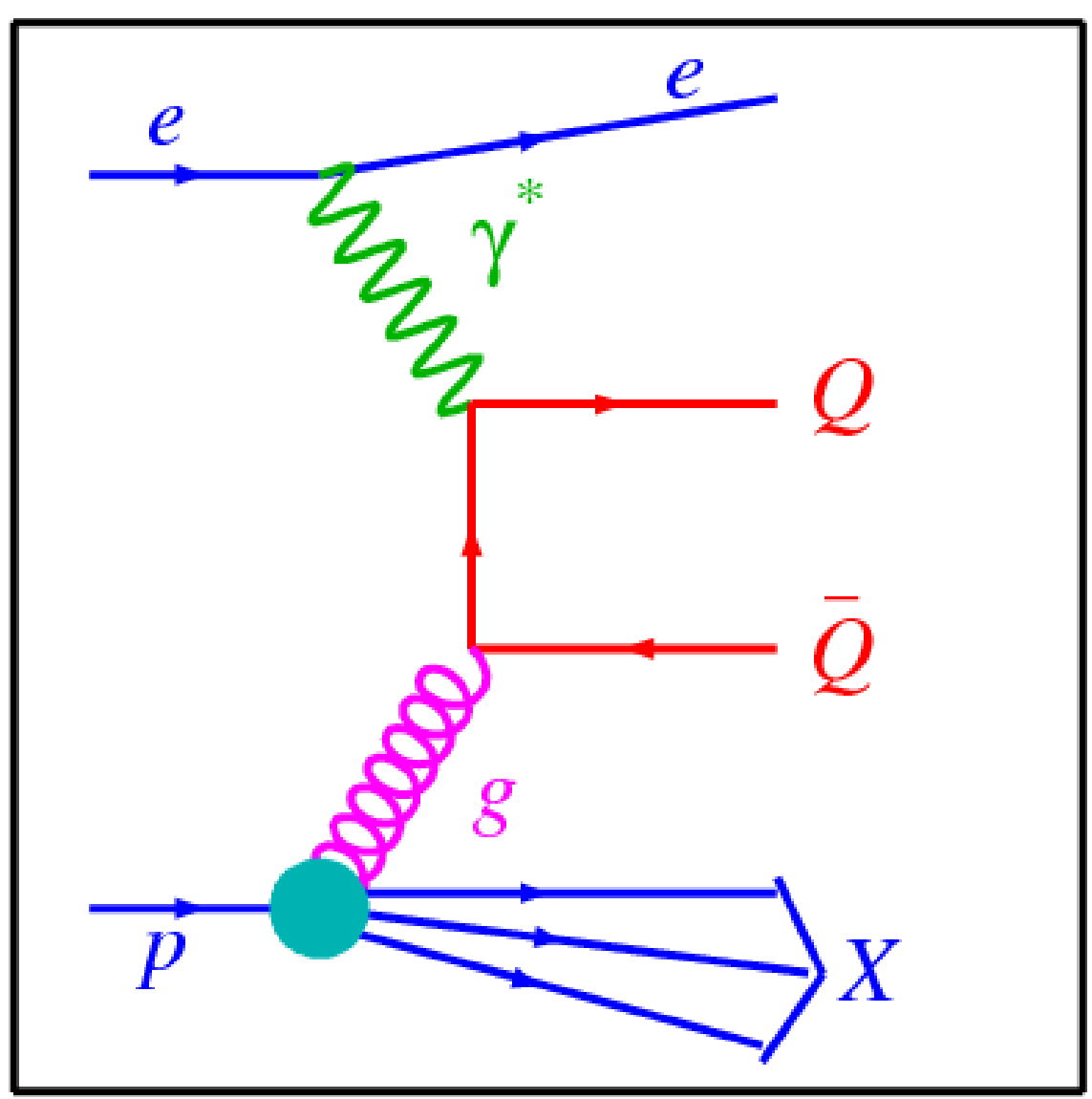




\section{Heavy Flavour Production at HERA}

- Life (QCD) is not quite so simple (2)

\section{Non-Direct}

Direct
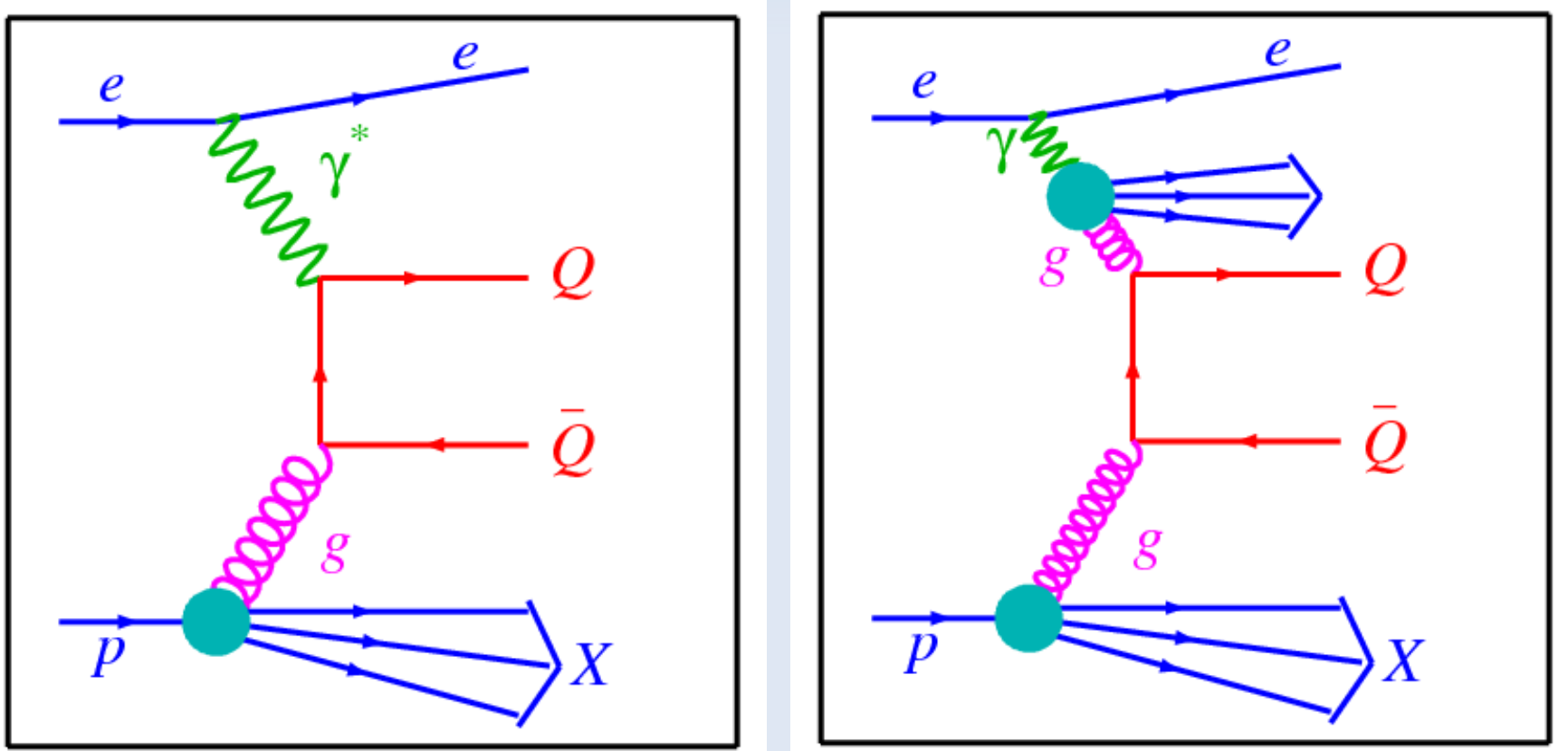
Ian C. Brock
Heavy Flavour Production at HERA

Resolved
Excitation

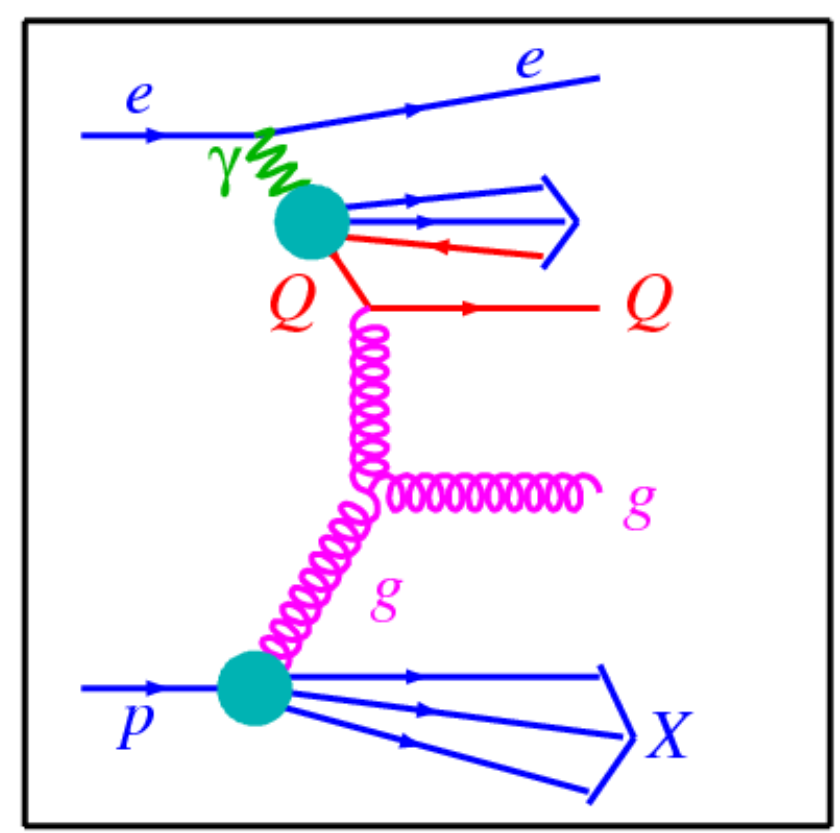

24/06/08 Slide 4 


\section{Heavy Flavour Production at HERA}

- HERA (ep):

- p: 920 (820) GeV

- e: $27.5 \mathrm{GeV}$

- $\mathrm{Q}^{2}=-\mathrm{q}^{2}=\left(\mathrm{k}-\mathrm{k}^{\prime}\right)^{2}$

- $\mathrm{Q}^{2}<1 \mathrm{GeV}^{2}$

- Photoproduction

- $\mathrm{Q}^{2}>1 \mathrm{GeV}^{2}$

- DIS

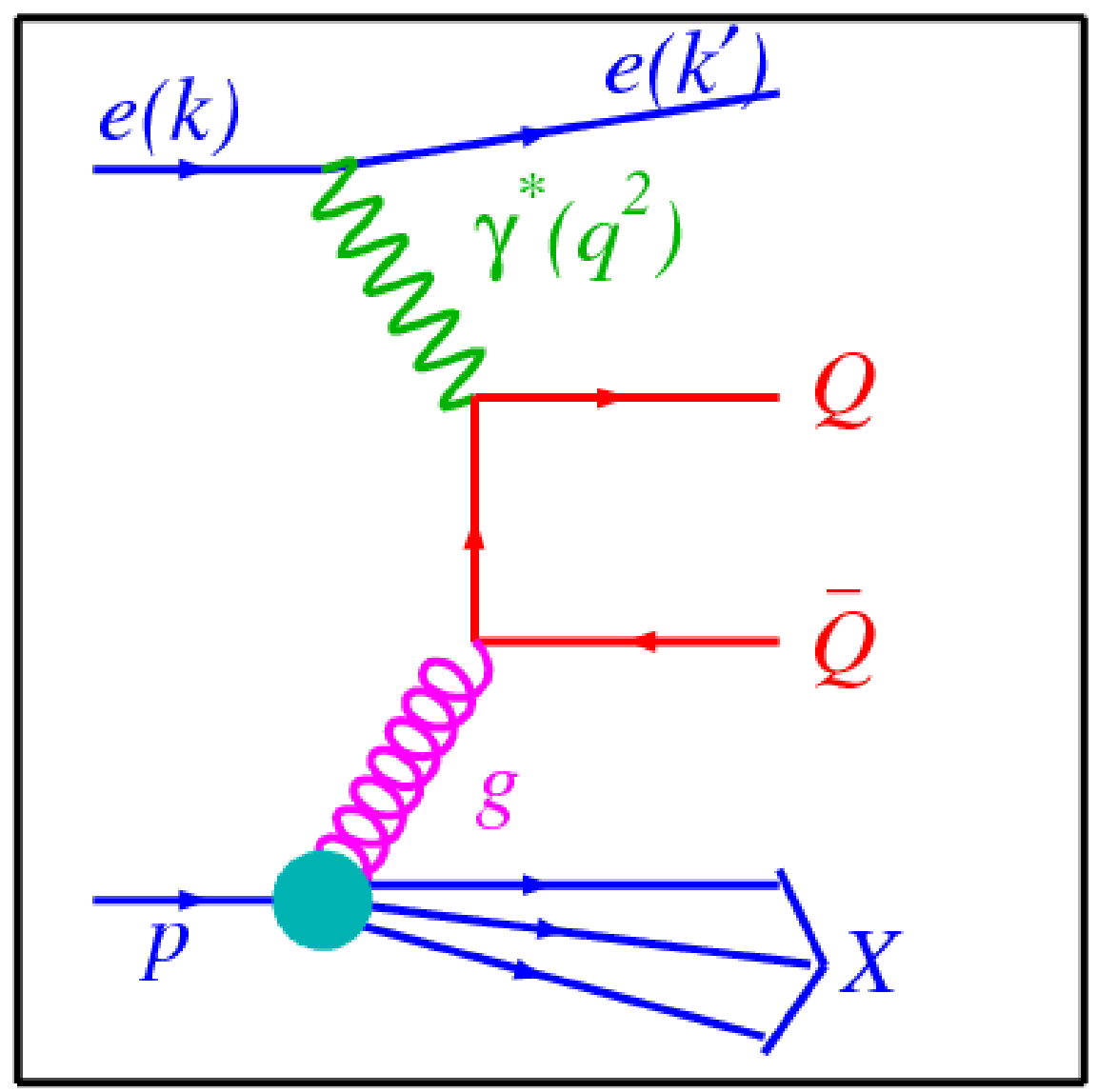




\section{Heavy Flavour Decay}

- Methods to tag HF:

- Reconstruct D* (or other D mesons)

- Tag semileptonic decay to $e, \mu$

- Use long B,D hadron lifetime

- Jet properites

- Different tags probe different kinematic regions

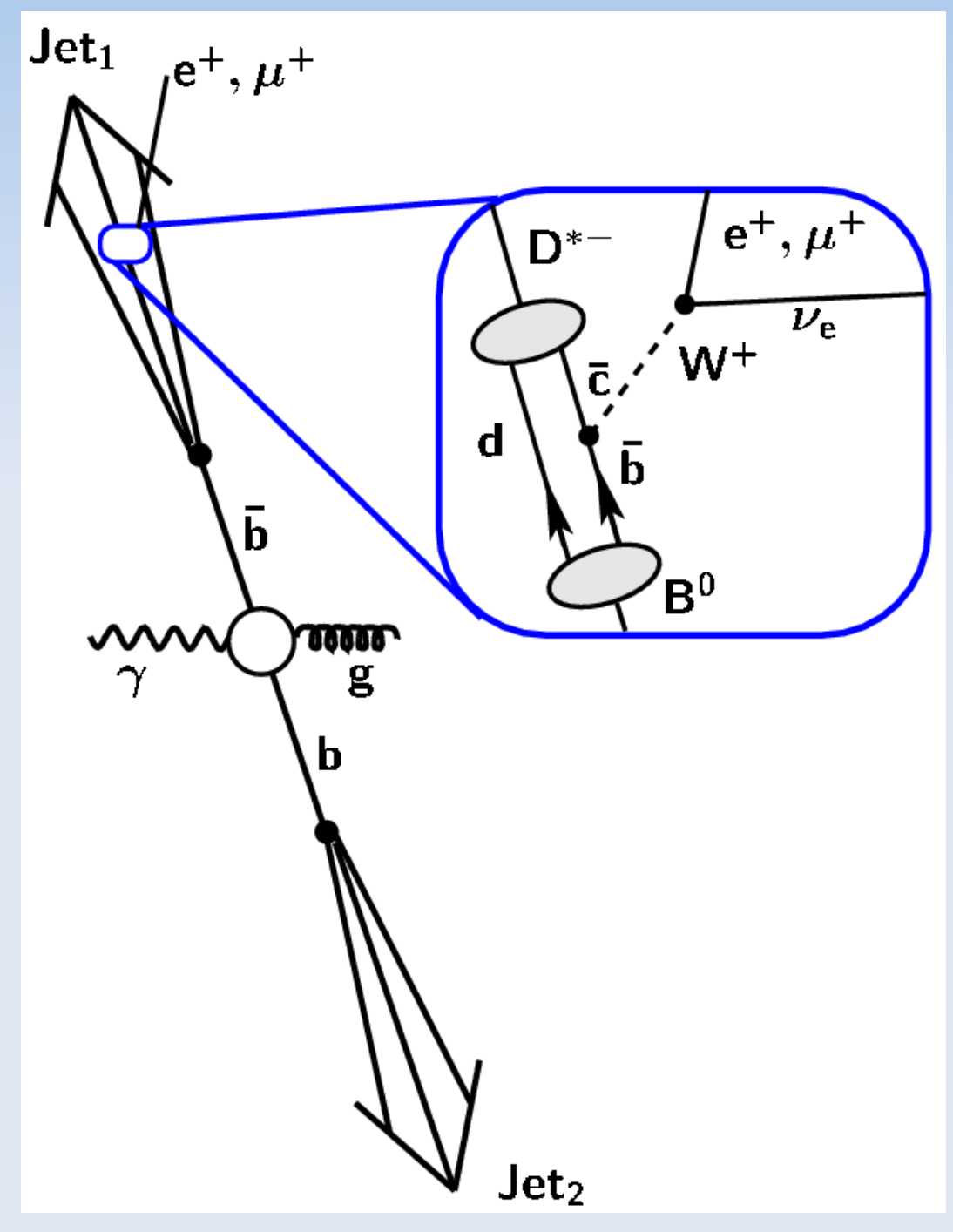




\section{The Theory}

- QCD Leading Order + - QCD NLO programs Parton Shower Monte Carlos

- PYTHIA, RAPGAP, HERWIG, CASCADE

- Massless \& massive matrix elements for charm

- Massive for beauty

- Used for acceptance corrections

- Weighted events

- Do not include parton shower

- FMNR for Photoproduction

- HVQDIS for DIS

- Usually compare with experiment by applying hadronic corrections from LO Monte Carlo 


\section{Luminosity \& Detectors}
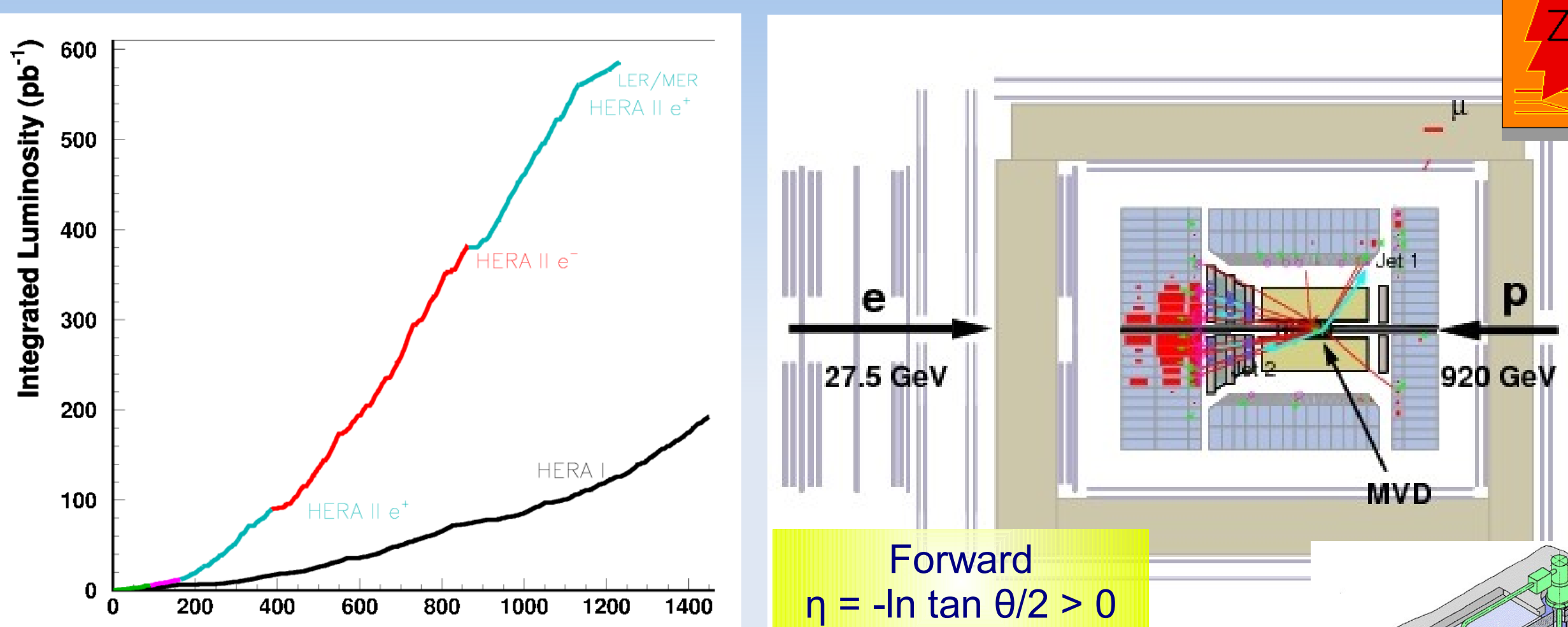

\section{Forward}

$\eta=-\ln \tan \theta / 2>0$

HERA I 1996-2000 HERA II 2004-2007

\section{Integrated luminosity on tape} $\sim 0.5 \mathrm{fb}^{-1}$ per experiment 


\section{Charm Production}

- HERA II data

- Two recent H1 analyses using new Fast Track Trigger

- Photoproduction

- $93 \mathrm{pb}^{-1}(2006 / 7)$

- DIS at low $Q^{2}$

- $247 \mathrm{pb}^{-1}(2004-7)$

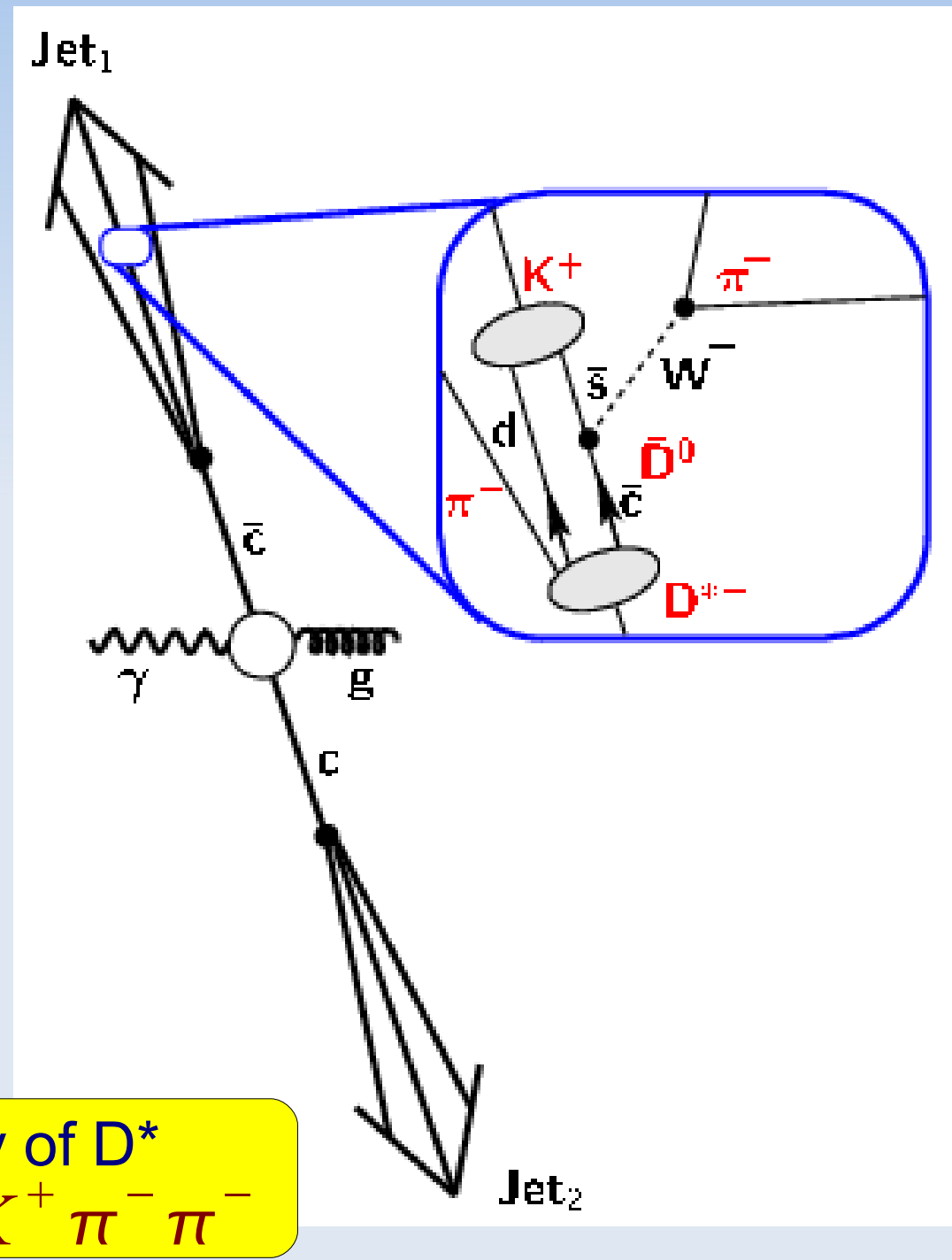

$$
\begin{gathered}
\text { "Golden" Decay of } \mathrm{D}^{*} \\
D^{*-} \rightarrow \bar{D}^{0} \pi^{-} \rightarrow K^{+} \pi^{-} \pi^{-}
\end{gathered}
$$




\section{Charm Production}

- Earlier ZEUS measurements include very low $Q^{2}$

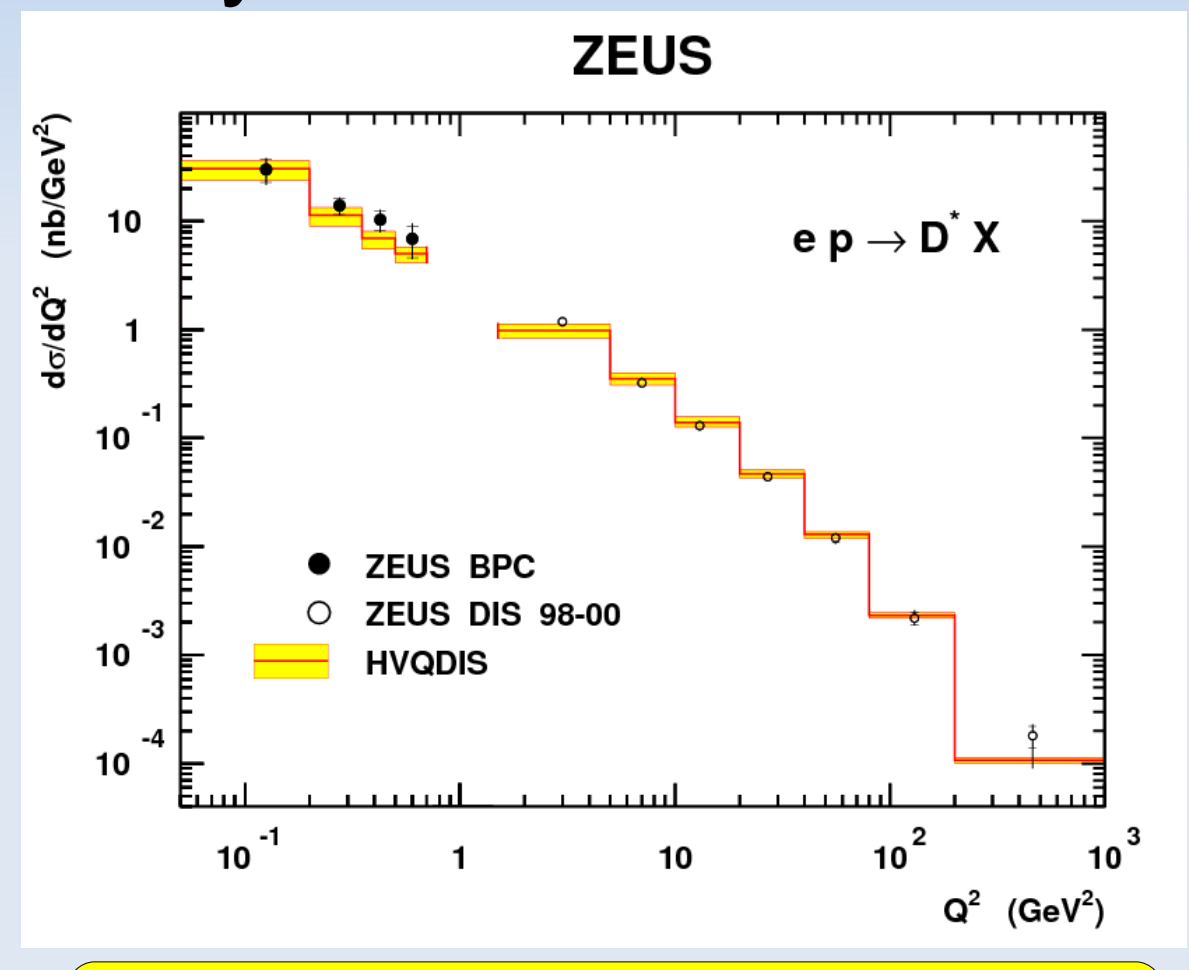

HVQDIS describes data over 5 orders of magnitude cross-section variation
- Single function to describe $y p$ crosssection over full $Q^{2}$ range

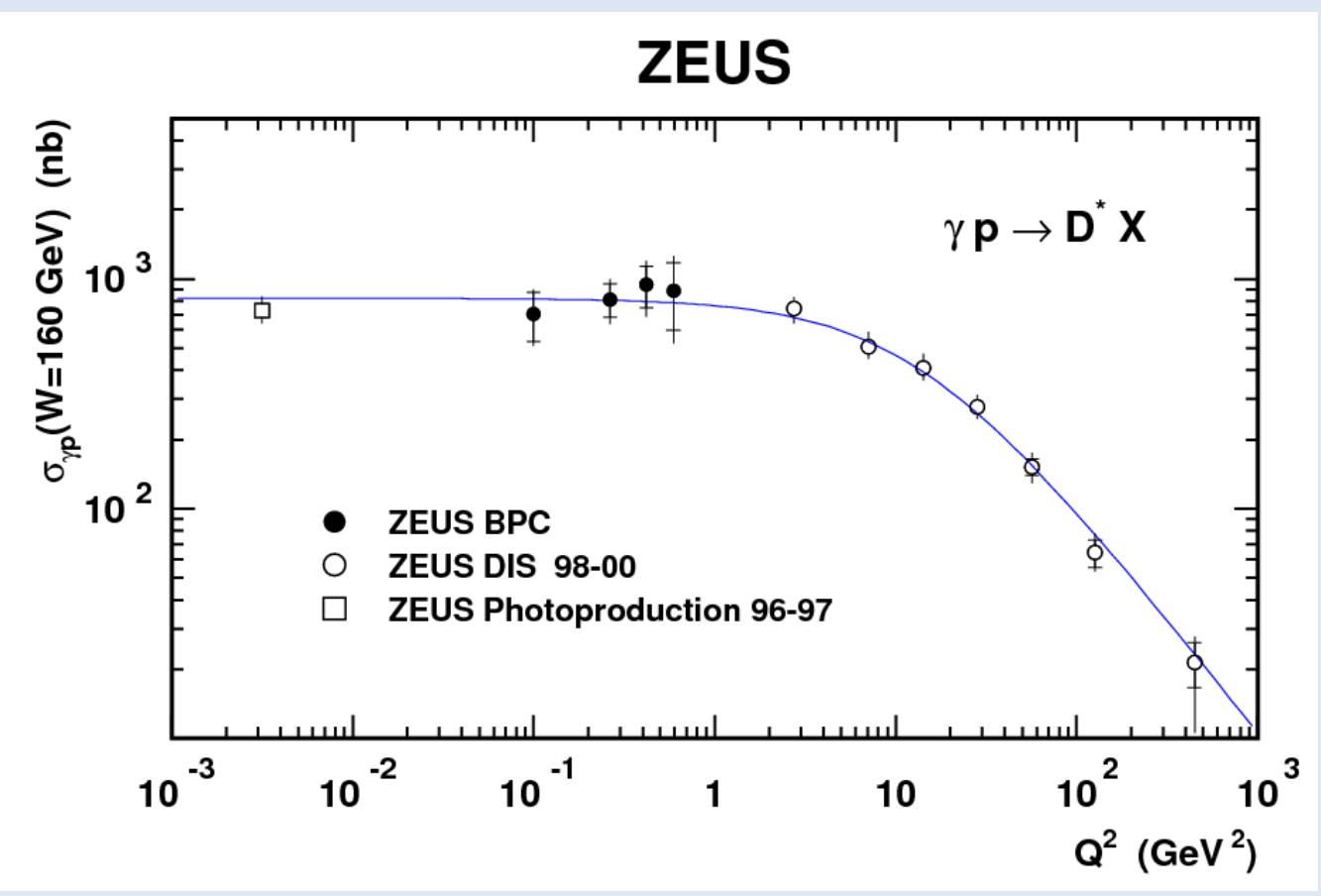




\section{Charm Production}

- Photoproduction:

\section{$\mathrm{Q}^{2}<2 \mathrm{GeV}^{2}$}

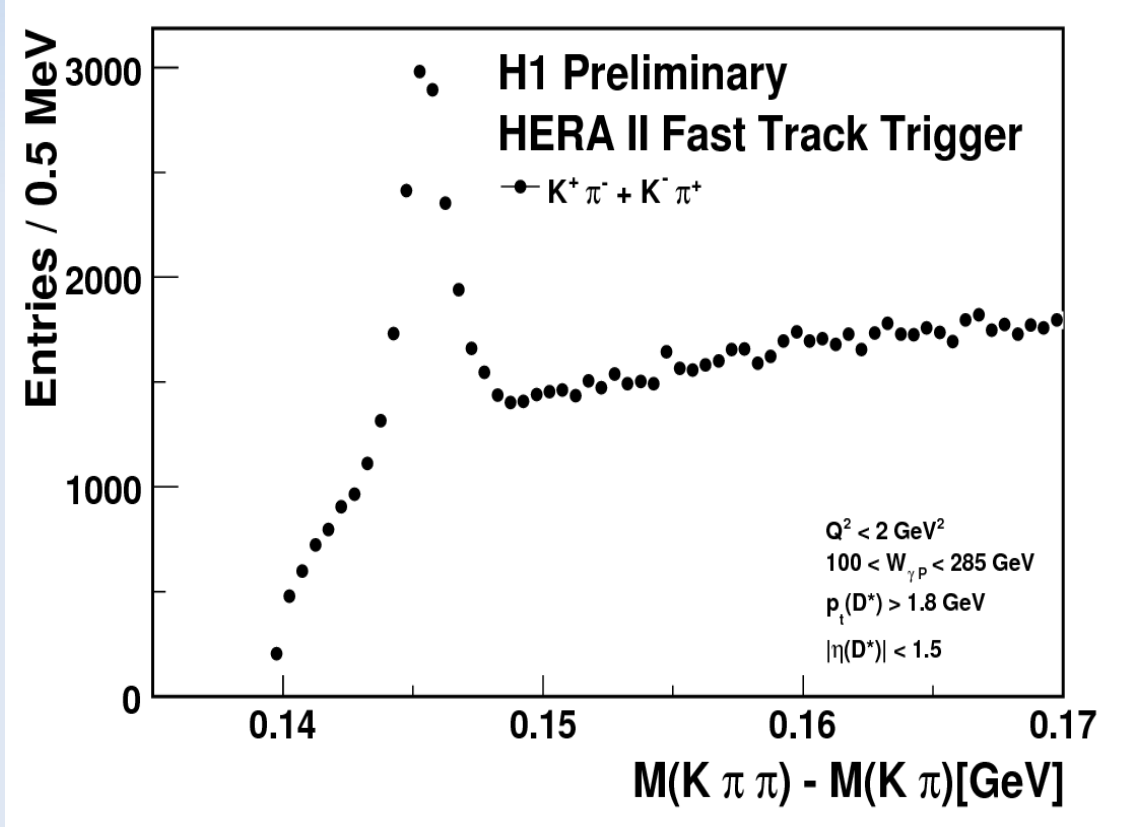

- DIS:

$$
\mathrm{Q}^{2}>5 \mathrm{GeV}^{2}
$$

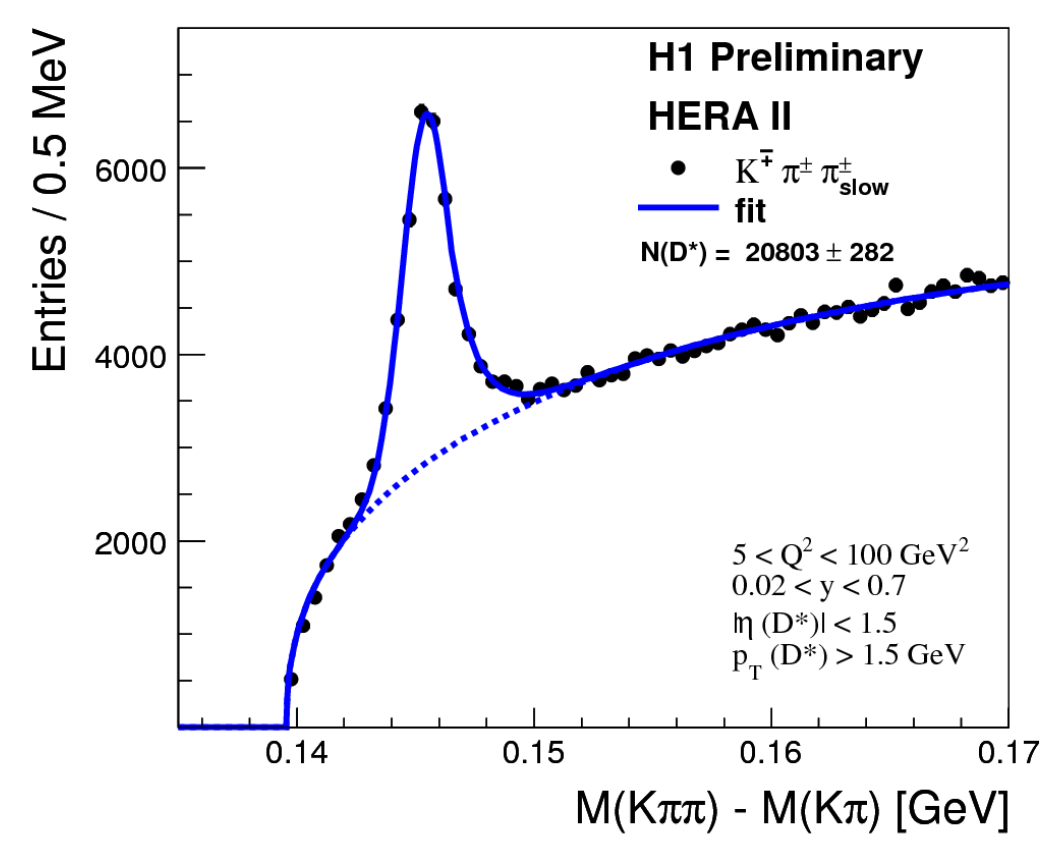

$$
D^{*-} \rightarrow \bar{D}^{0} \pi^{-} \rightarrow K^{+} \pi^{-} \pi^{-}
$$




\section{Charm Production}

- Cross-section as a function of

- $Q^{2}$

- $\mathrm{P}_{\mathrm{T}}, \eta$ of $\mathrm{D}^{*}$

- Photoproduction:

- W (yp CM energy)

- DIS

- y (inelasticity)

- Compared to MC and NLO predictions

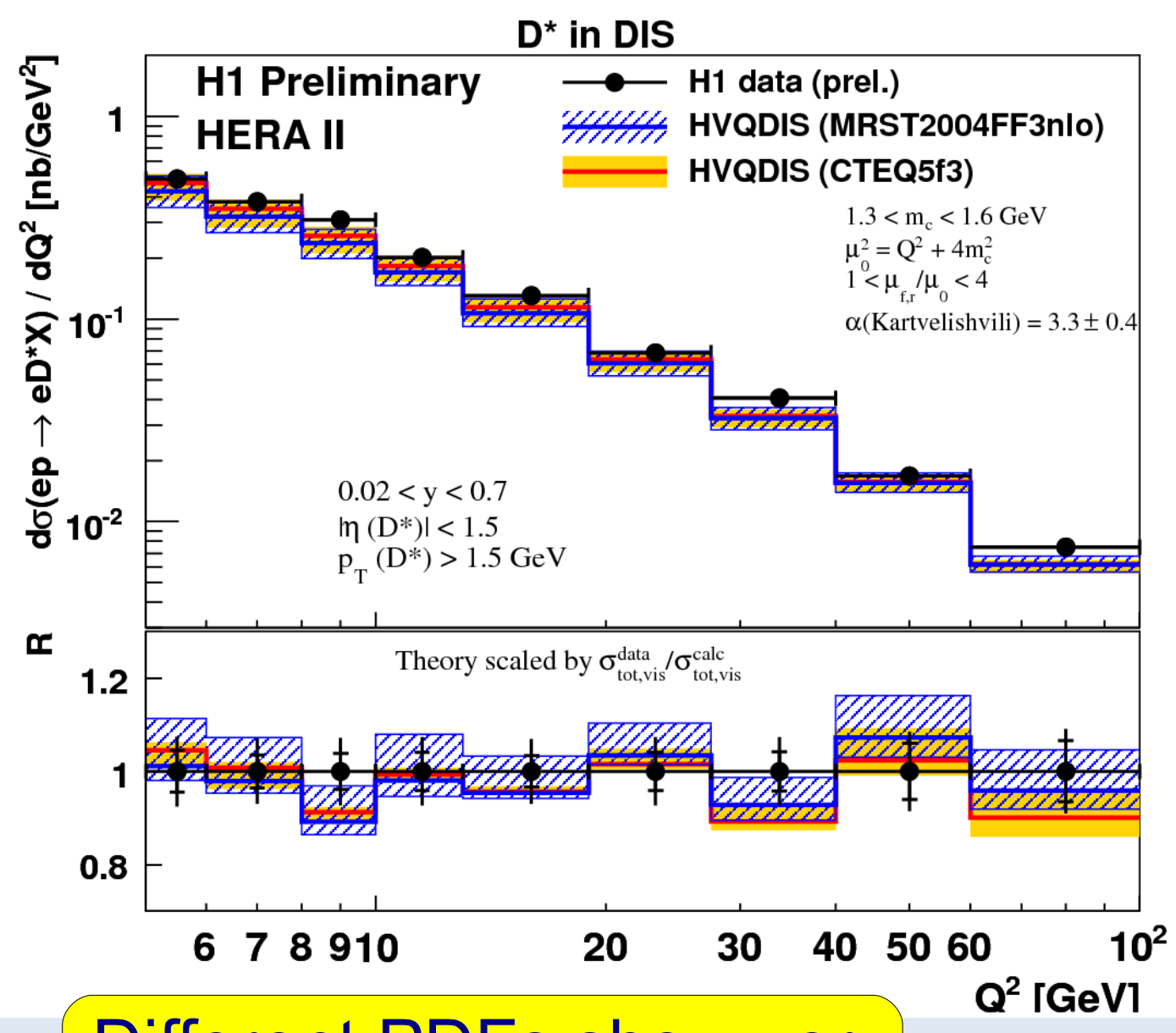

Different PDFs show very similar $Q^{2}$ behaviour 


\section{Charm in DIS}

\section{- Compare with MC}

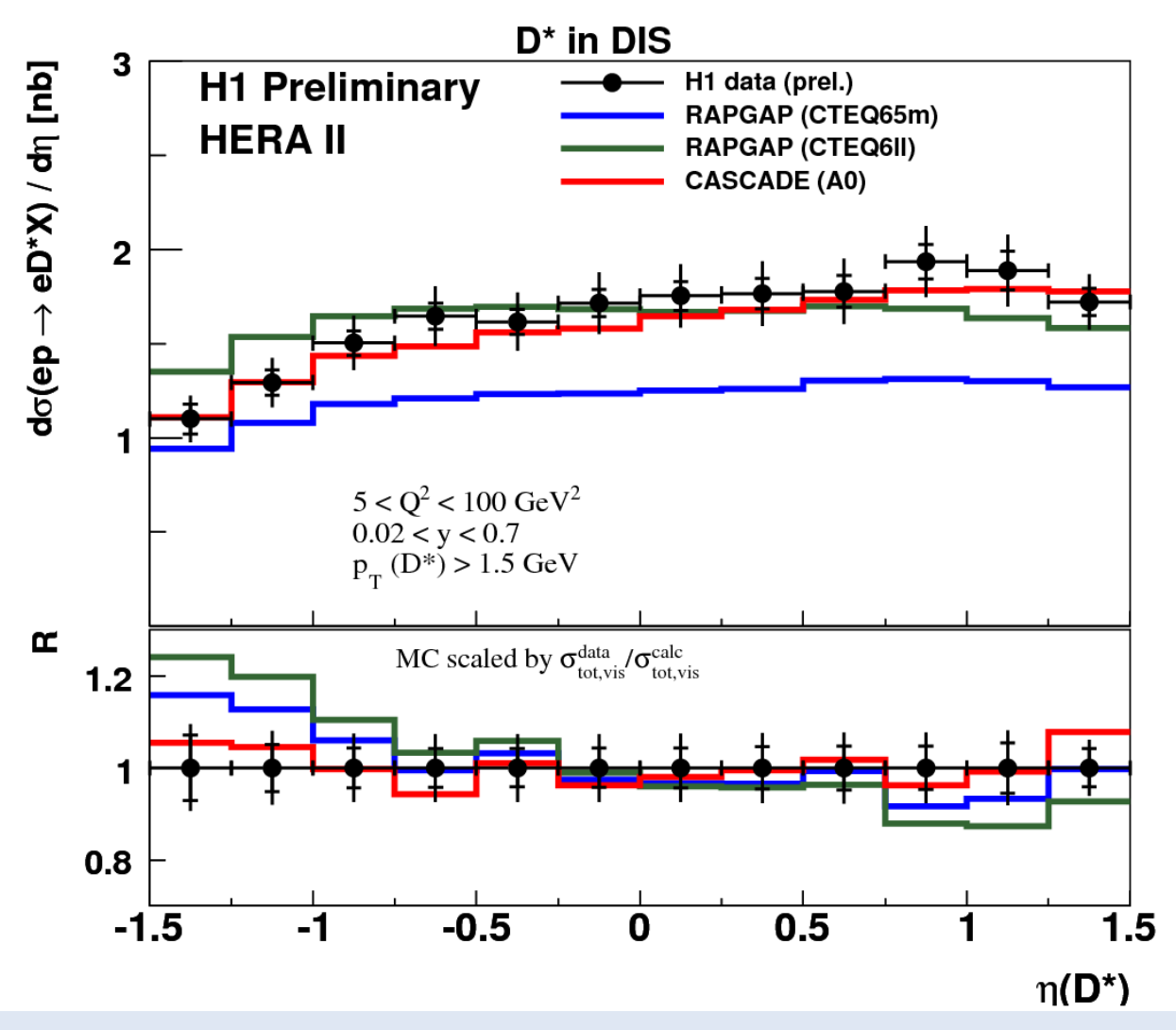

Significant changes for different PDFs Cascade agrees very well with data

\section{- Compare with NLO}

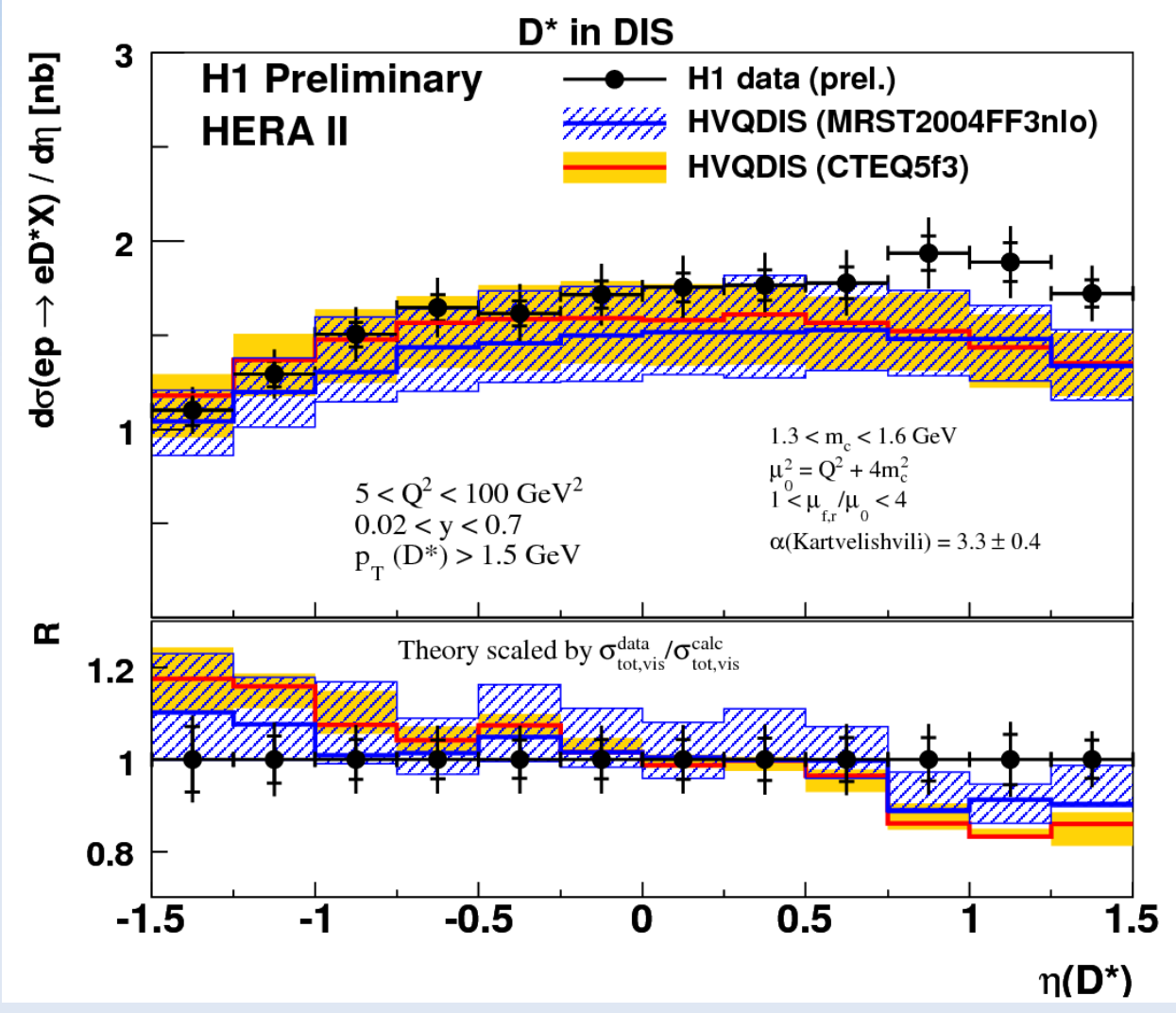

Data overshoot prediction at large $\eta$ Some sensitivity to gluon: MRST vs CTEQ
Heavy Flavour Production at HERA Ian C. Brock
$24 / 06 / 08$ Slide 13 


\section{Charm in Photoproduction}

\section{- Compare with MC}
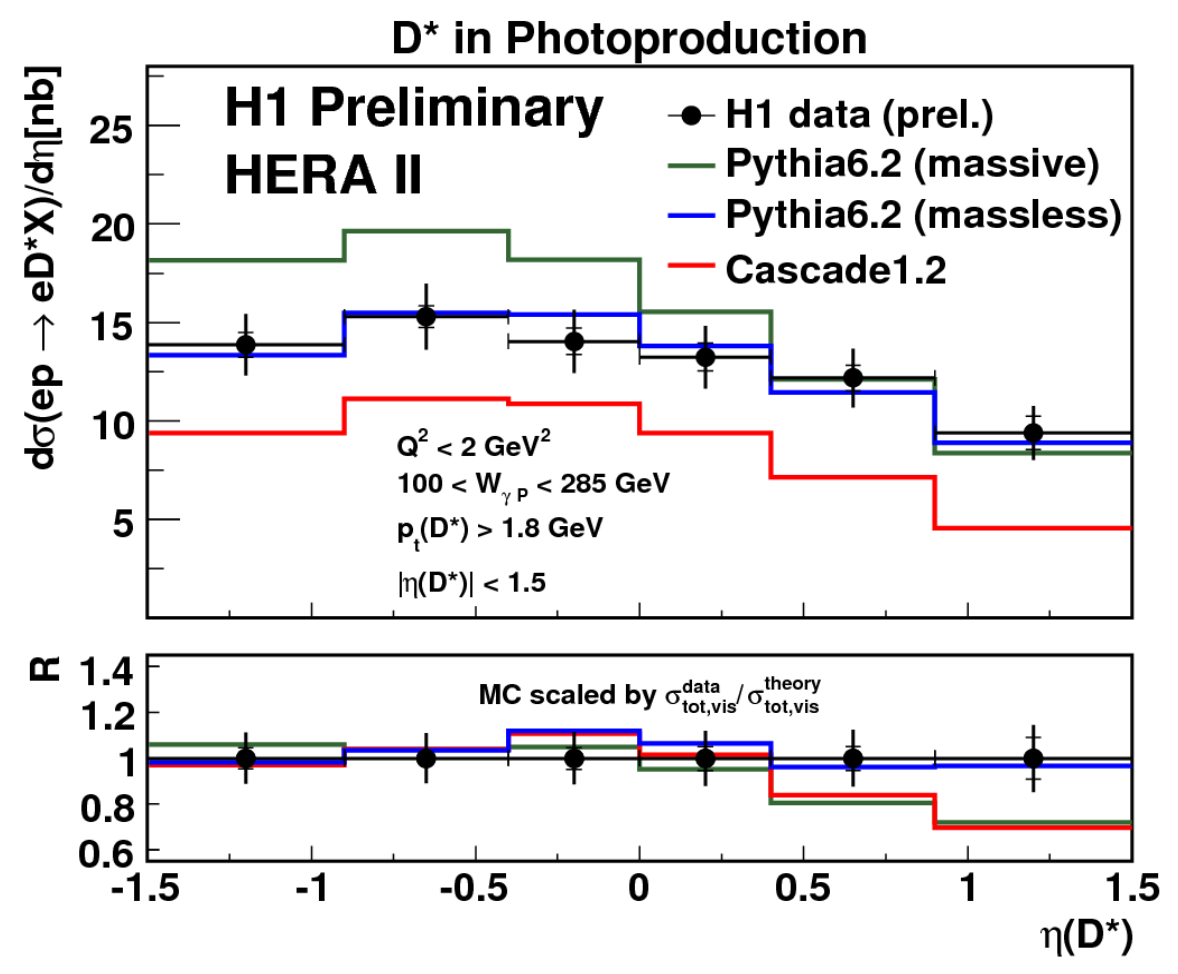

Significant changes for different MCs

Pythia with massless charm agrees very well with data

\section{- Compare with NLO}

\section{$D^{*}$ in Photoproduction}

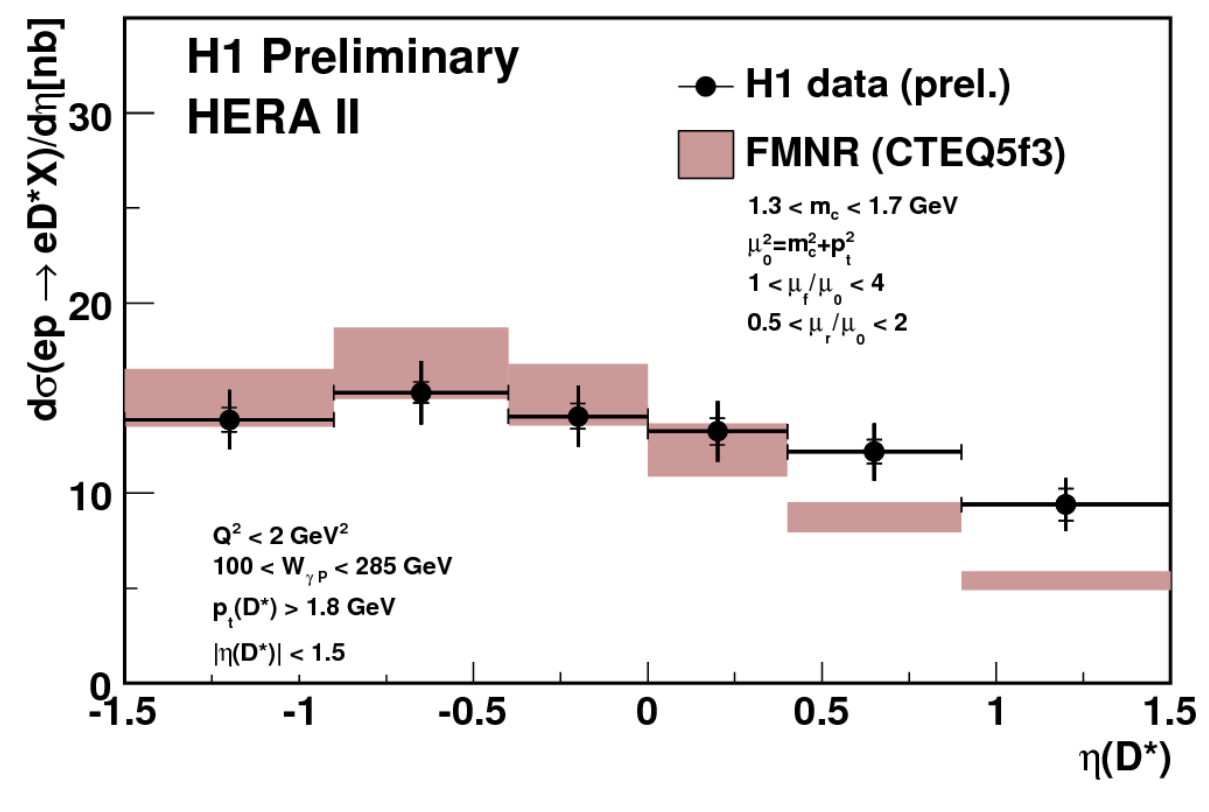

Data overshoot prediction at large $\eta$
Heavy Flavour Production at HERA Ian C. Brock 


\section{Beauty in Photoproduction}

- HERA II data

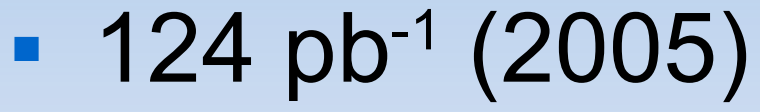

- Photoproduction

- Dijet events

- $P_{T}^{\text {jet }}>7(6) \mathrm{GeV}$

- Semileptonic decays to muons $\left(p_{T}^{\mu}>2.5 \mathrm{GeV}\right)$

- Include lifetime information

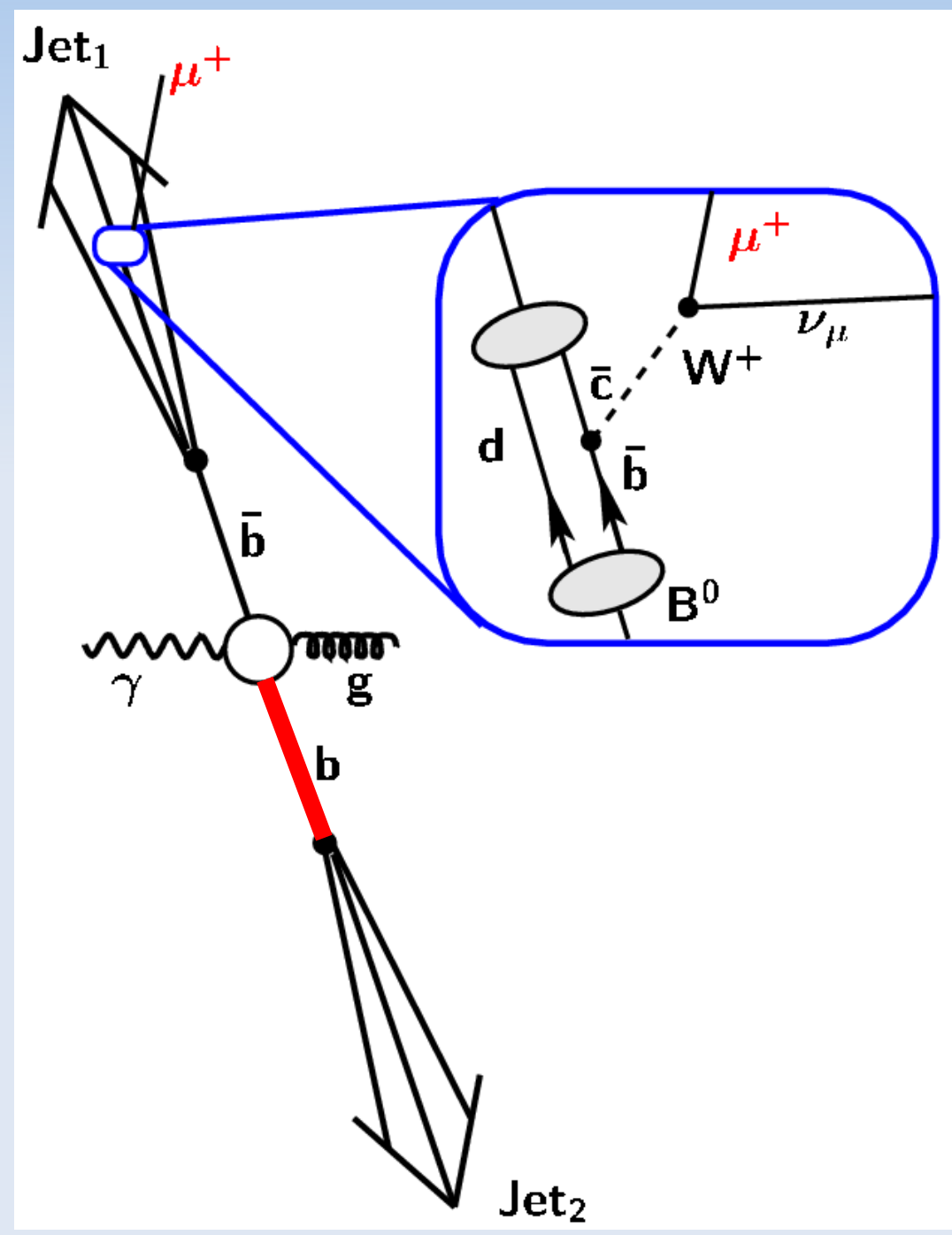




\section{Beauty in Photoproduction}

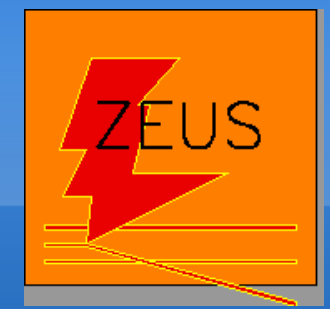

- $p_{T}{ }^{r e l}$
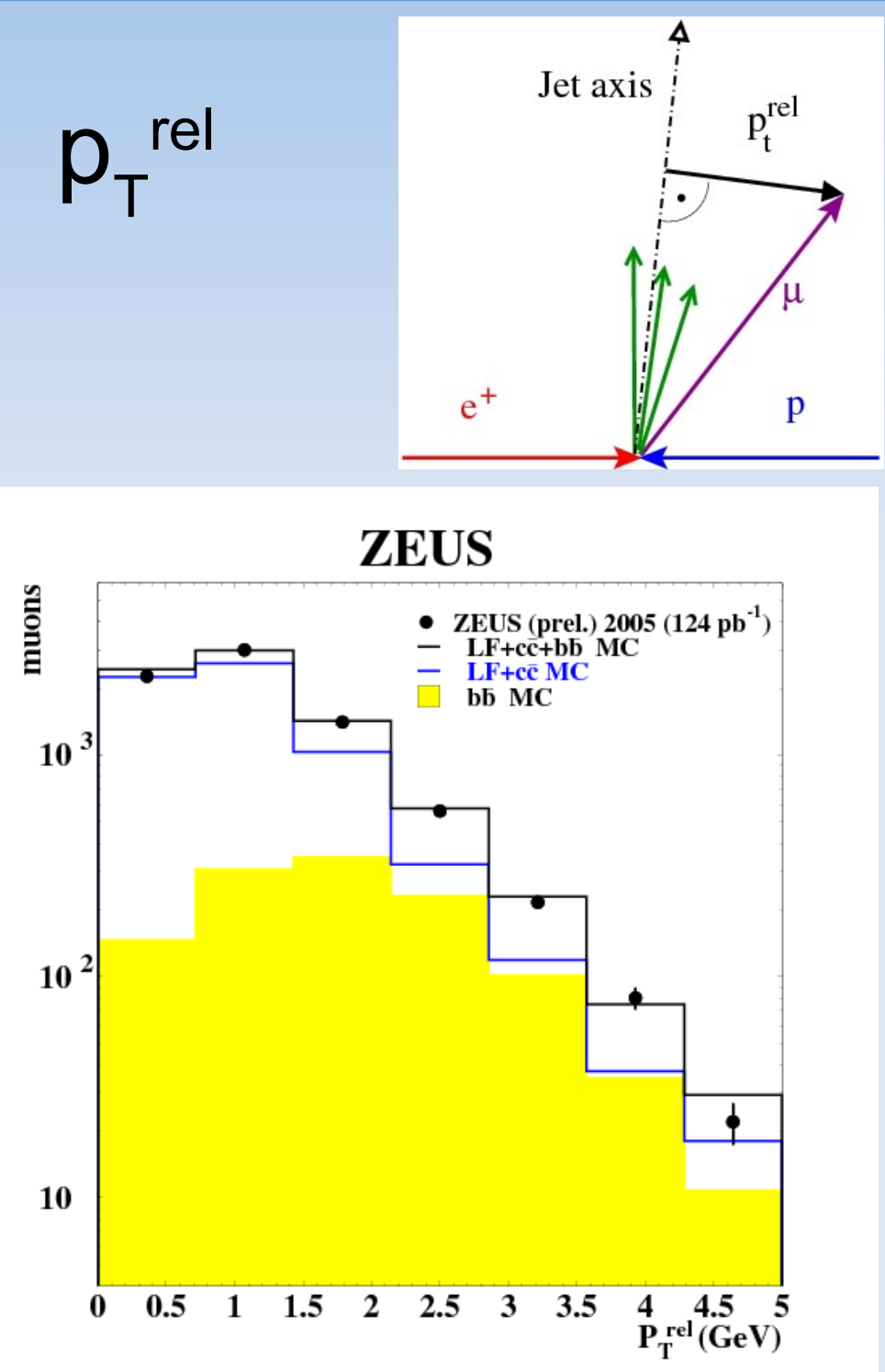

- Impact parameter

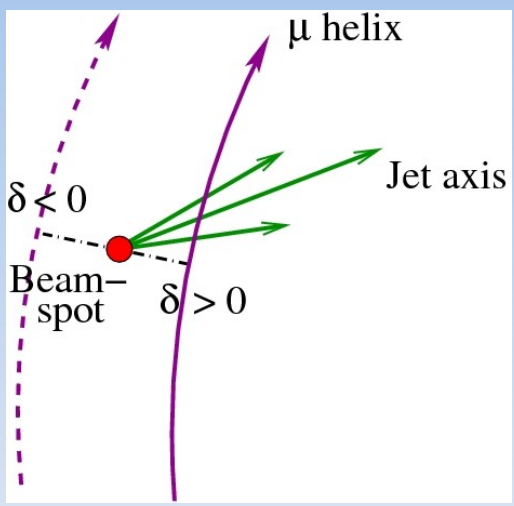

\section{ZEUS}

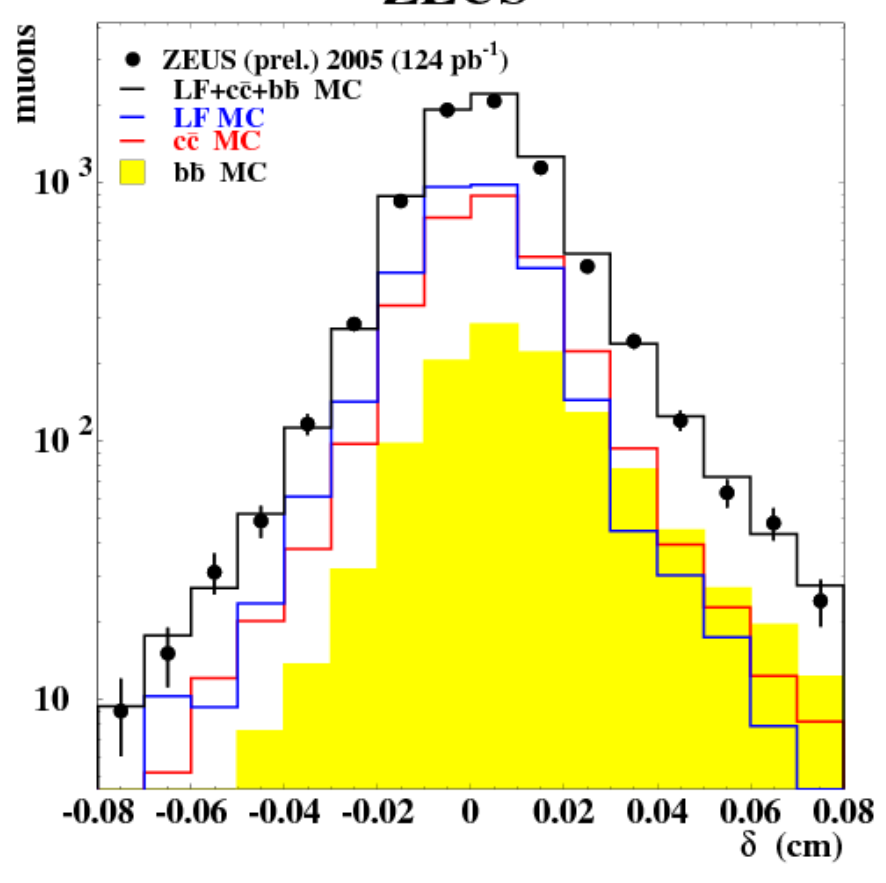




\section{Beauty in Photoproduction}

$$
\begin{aligned}
\sigma^{\text {vis }} & =46.8 \pm 4.0(\text { stat. })_{-7.2}^{+6.1}(\text { syst. }) \mathrm{pb} \\
\sigma^{N L O} & =41.5_{-8.9}^{+13.9} \mathrm{pb} \begin{array}{l}
\text { Renormalisation/factorisation scales } \\
\text { b quark mass }
\end{array}
\end{aligned}
$$
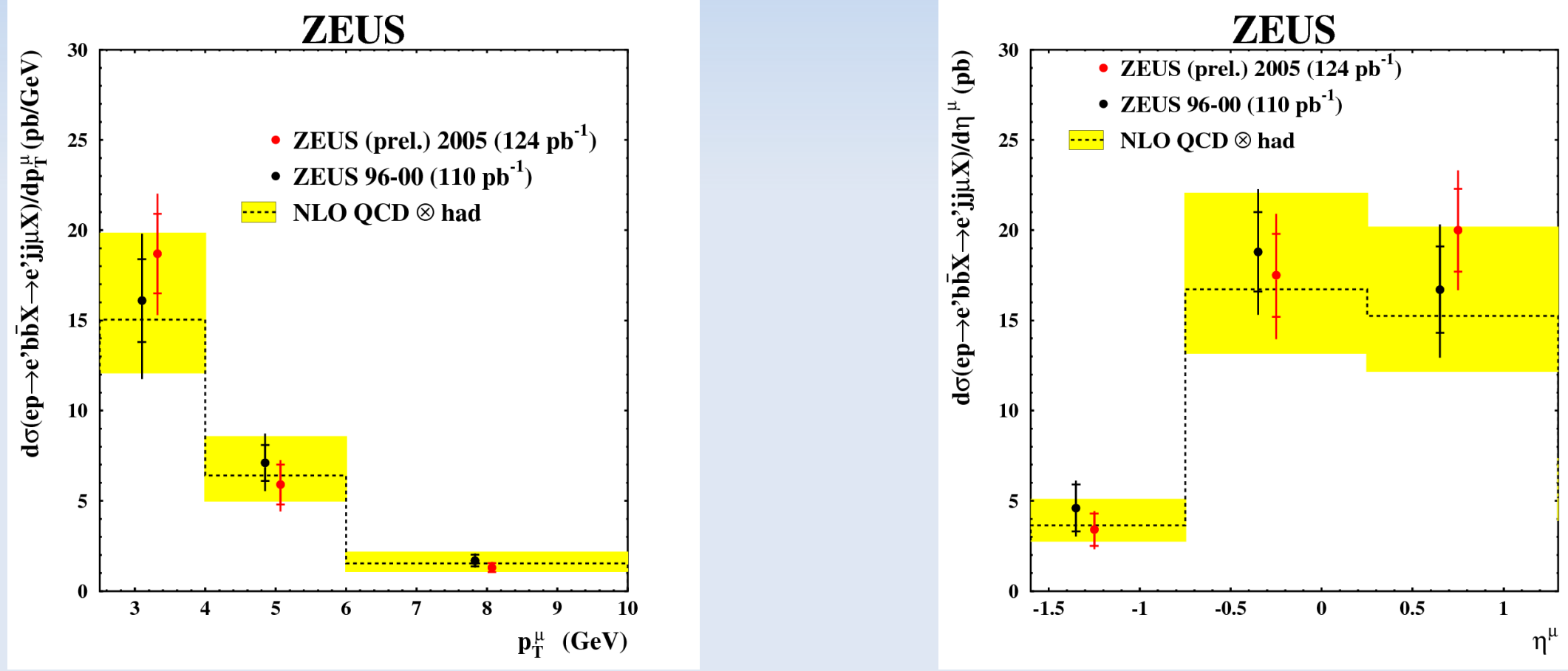

$\mathrm{P}_{\mathrm{T}}{ }^{\mu}>2.5 \mathrm{GeV}$

\section{Good agreement between} HERA I and HERA II cross-sections 


\section{b \& c in Photoproduction}

- HERA I data

- $120 \mathrm{pb}^{-1}(1996-2000)$ Bkg

- Dijet photoproduction events

$$
\text { - } E_{T}^{\text {jet }}>7(6) \mathrm{GeV}
$$

- Semileptonic decays to electrons $\left(p_{T}{ }^{e}>0.9 \mathrm{GeV}\right)$

- Look for more variables to determine $\mathrm{b}$ and $\mathrm{c}$ quark fractions separately

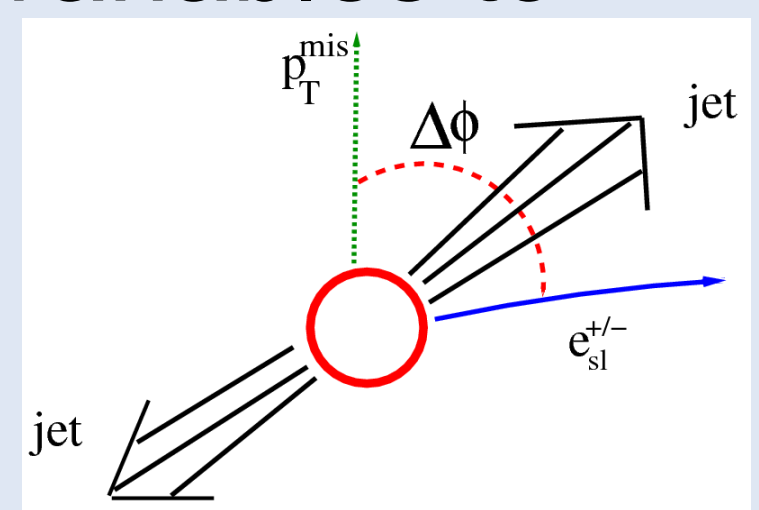

Heavy Flavour Production at HERA Ian C. Brock
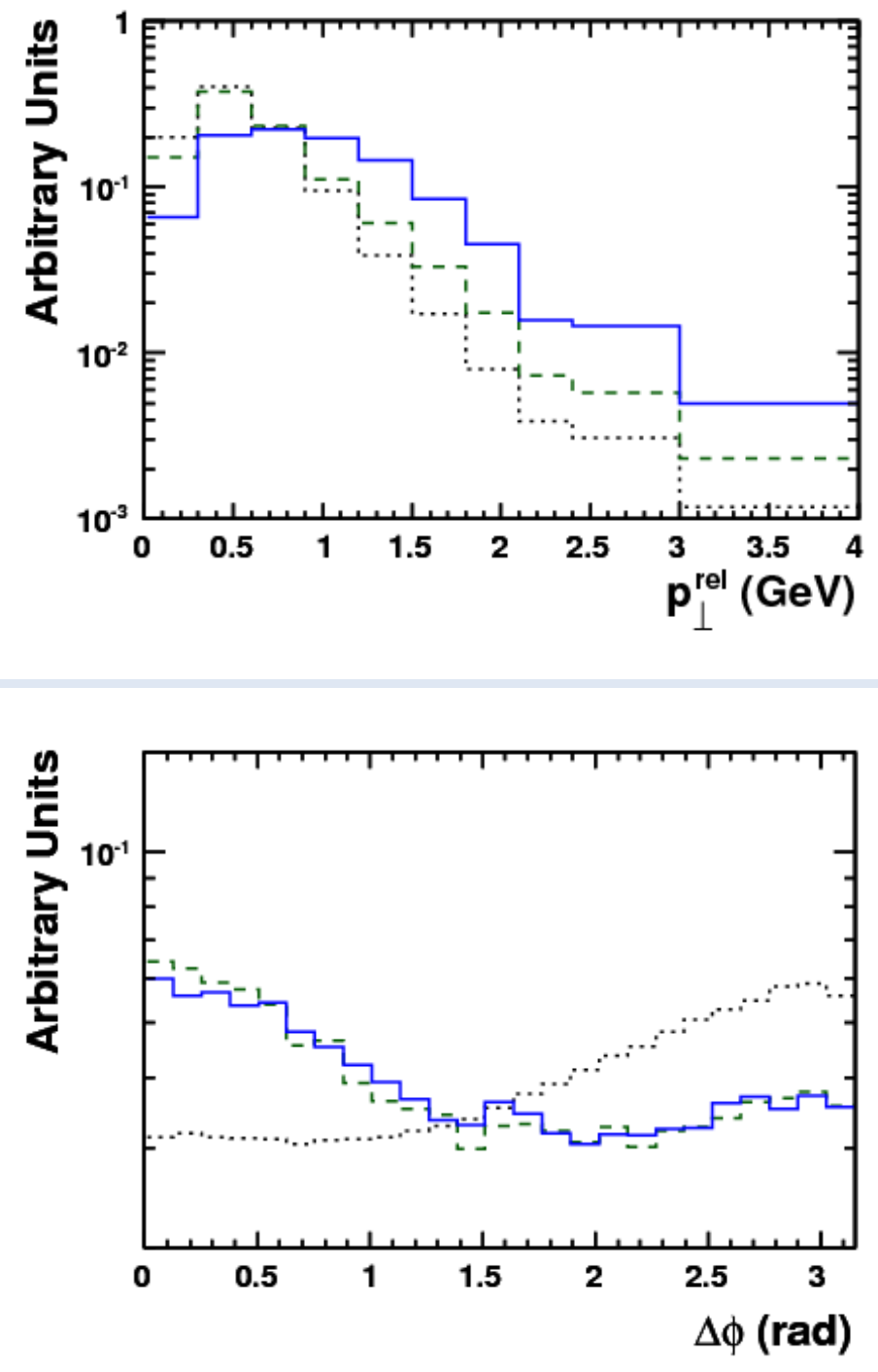


\section{b \& c in Photoproduction}

- Use a likelihood ratio method to separate b,c and light flavour

\section{ZEUS}

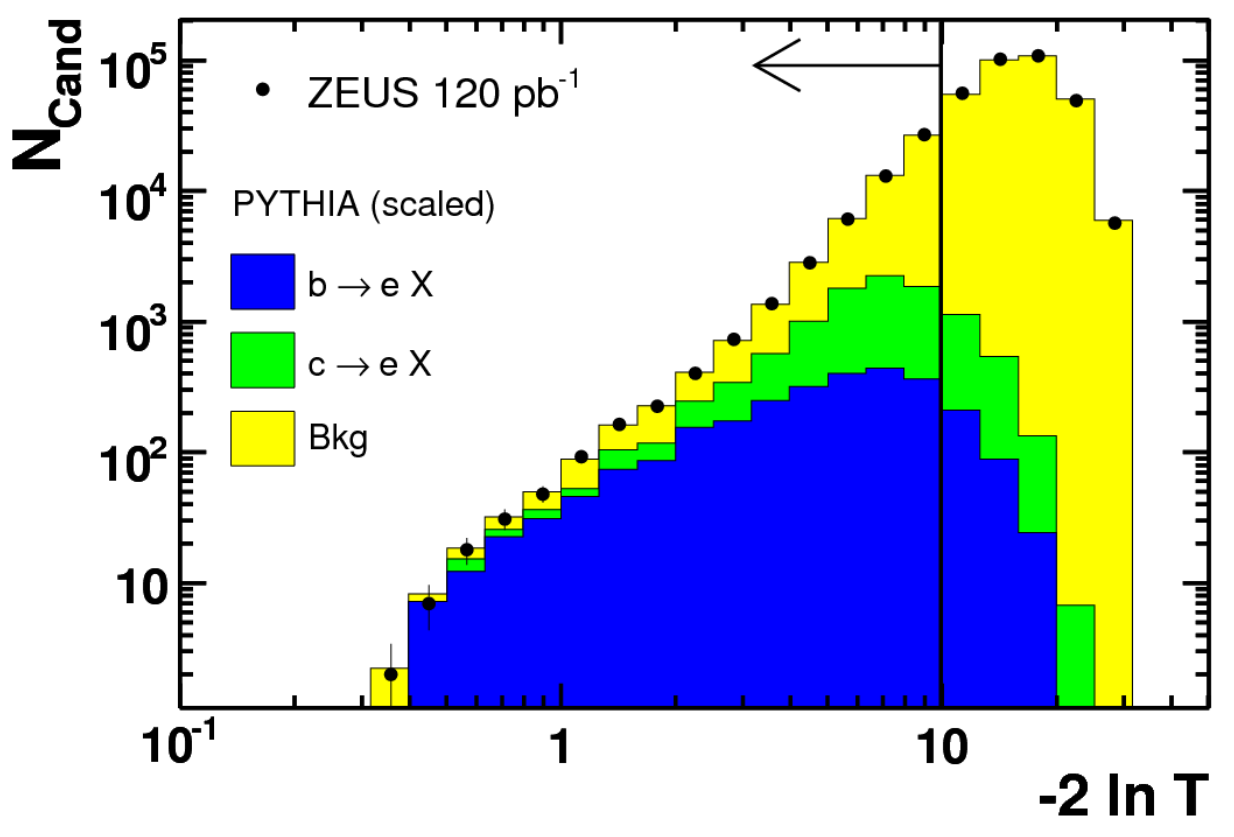

ZEUS

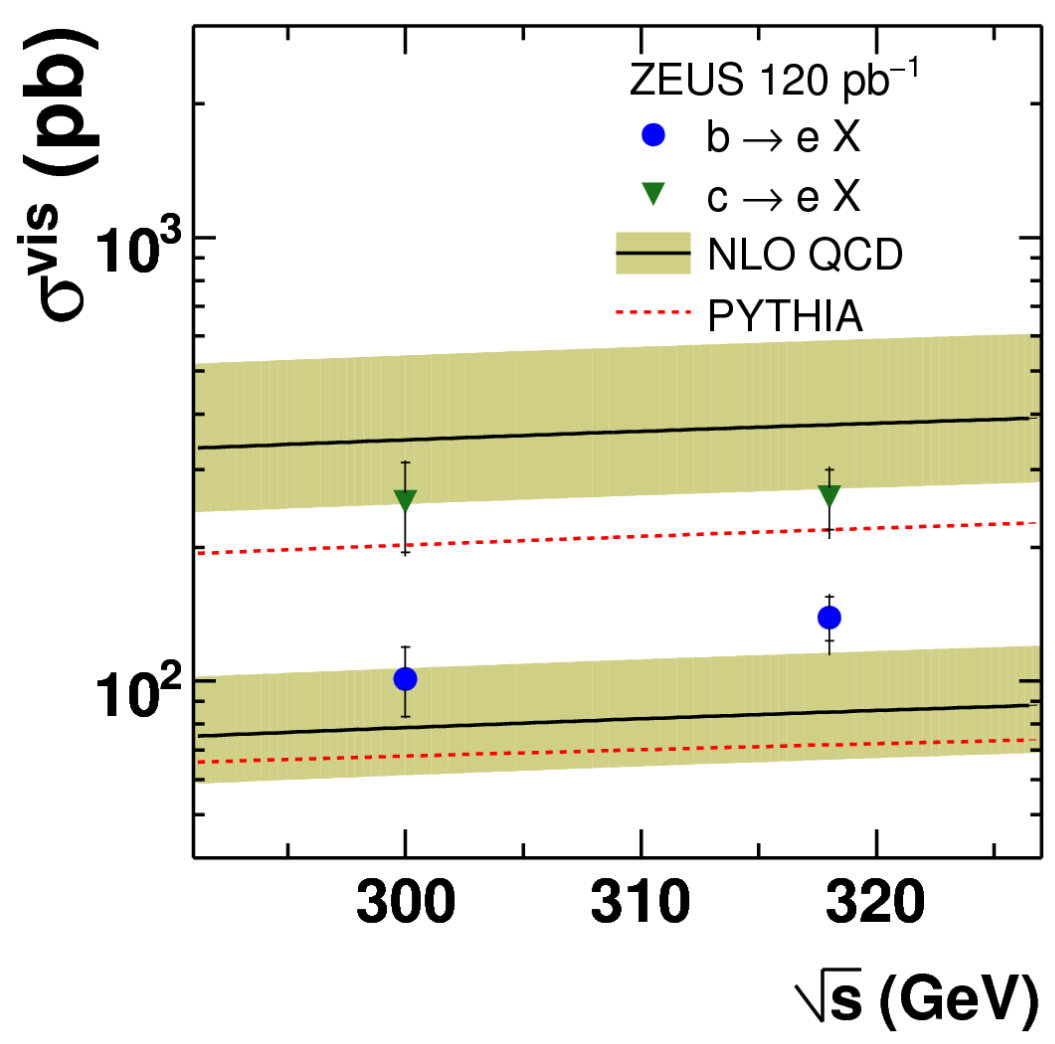

Heavy Flavour Production at HERA Ian C. Brock
24/06/08 Slide 19 


\section{b \& c in Photoproduction}

\section{Beauty}

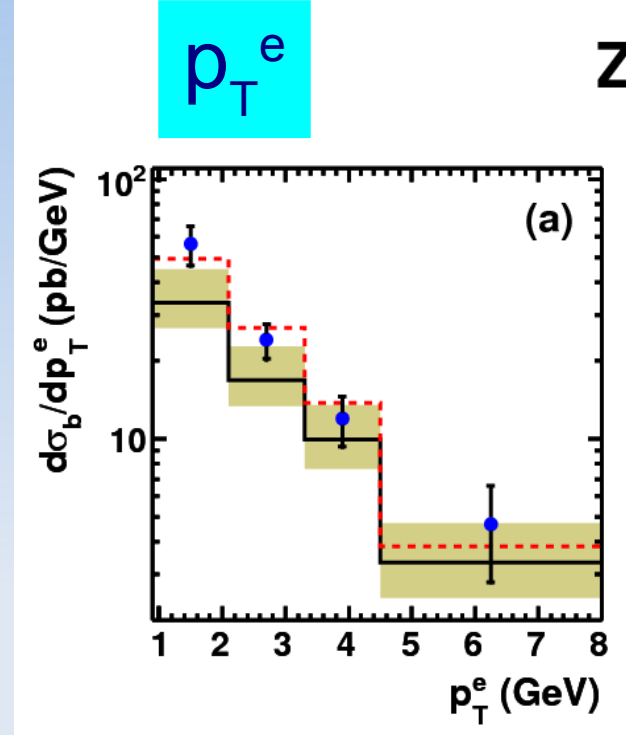

\section{ZEUS}
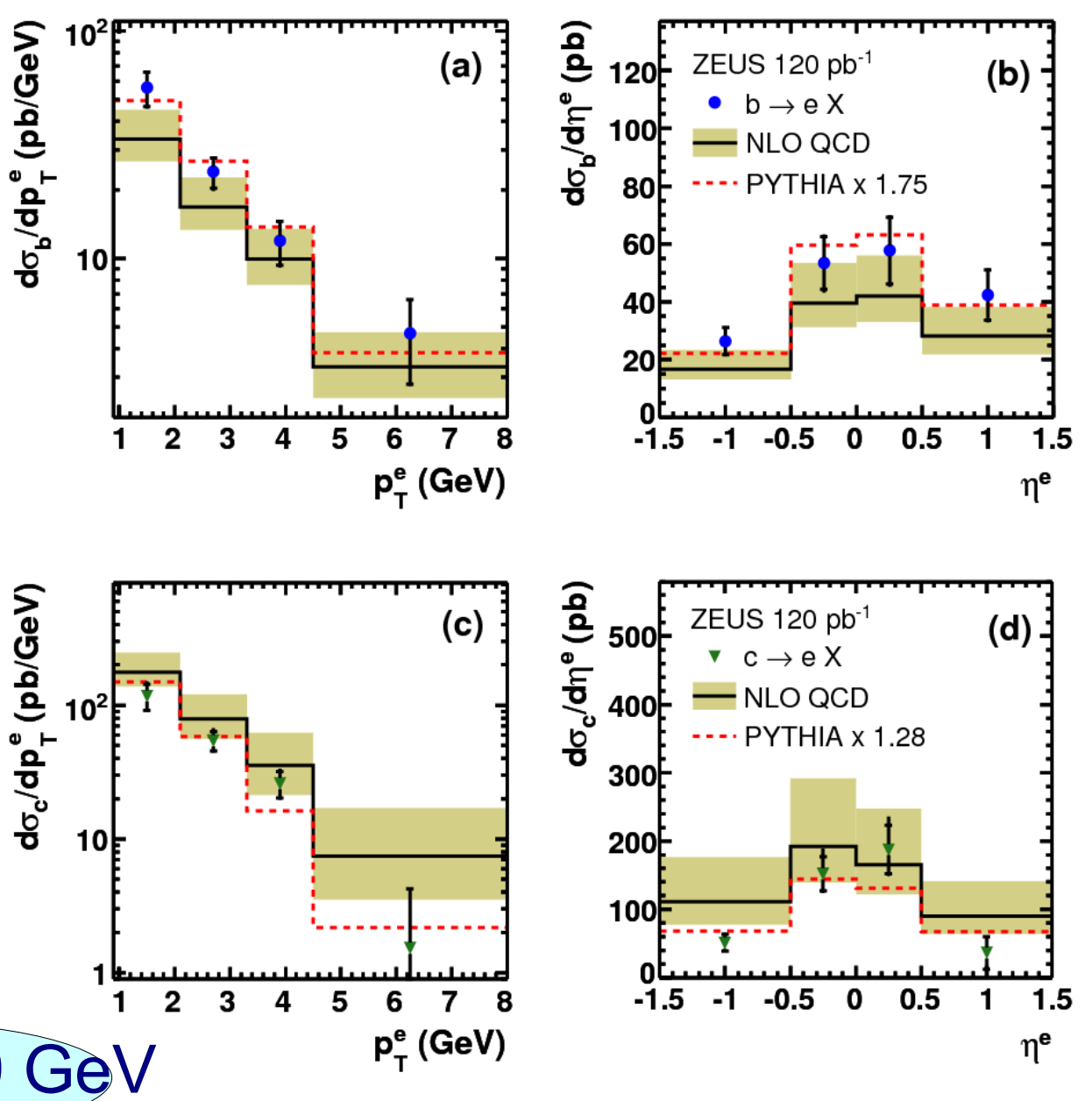

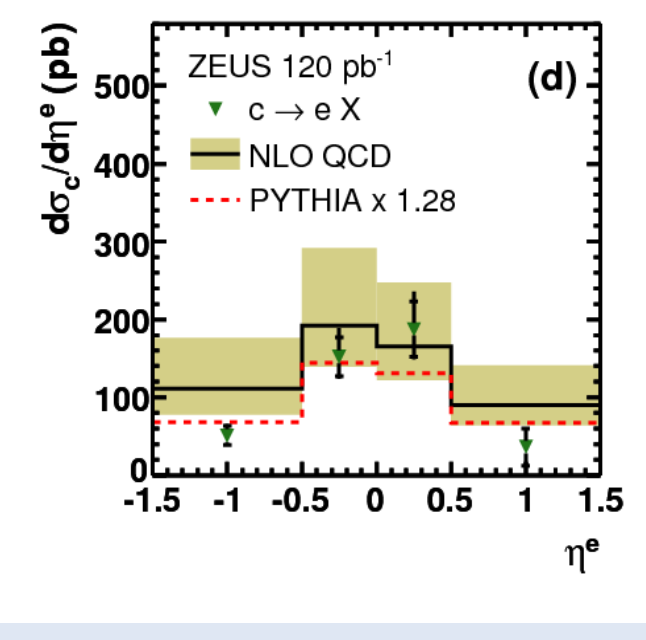

- LO Monte Carlo scale factors:

- bx 1.75

- c X 1.28

- NLO absolute predictions

\section{Charm}

$P_{\mathrm{T}}{ }^{e}>0.9 \mathrm{GeV}$

Heavy Flavour Production at HERA Ian C. Brock 


\section{b $\bar{b}$ Production}

- Double tag events

- Low background $\odot$

- Larger kinematic range $\odot$

- Low statistics $\odot$

- $\mathrm{E}_{\mathrm{T}}>8 \mathrm{GeV}$

- Two identified muons

- PhP + DIS

- Measure b̄ correlations

- Probe NLO effects

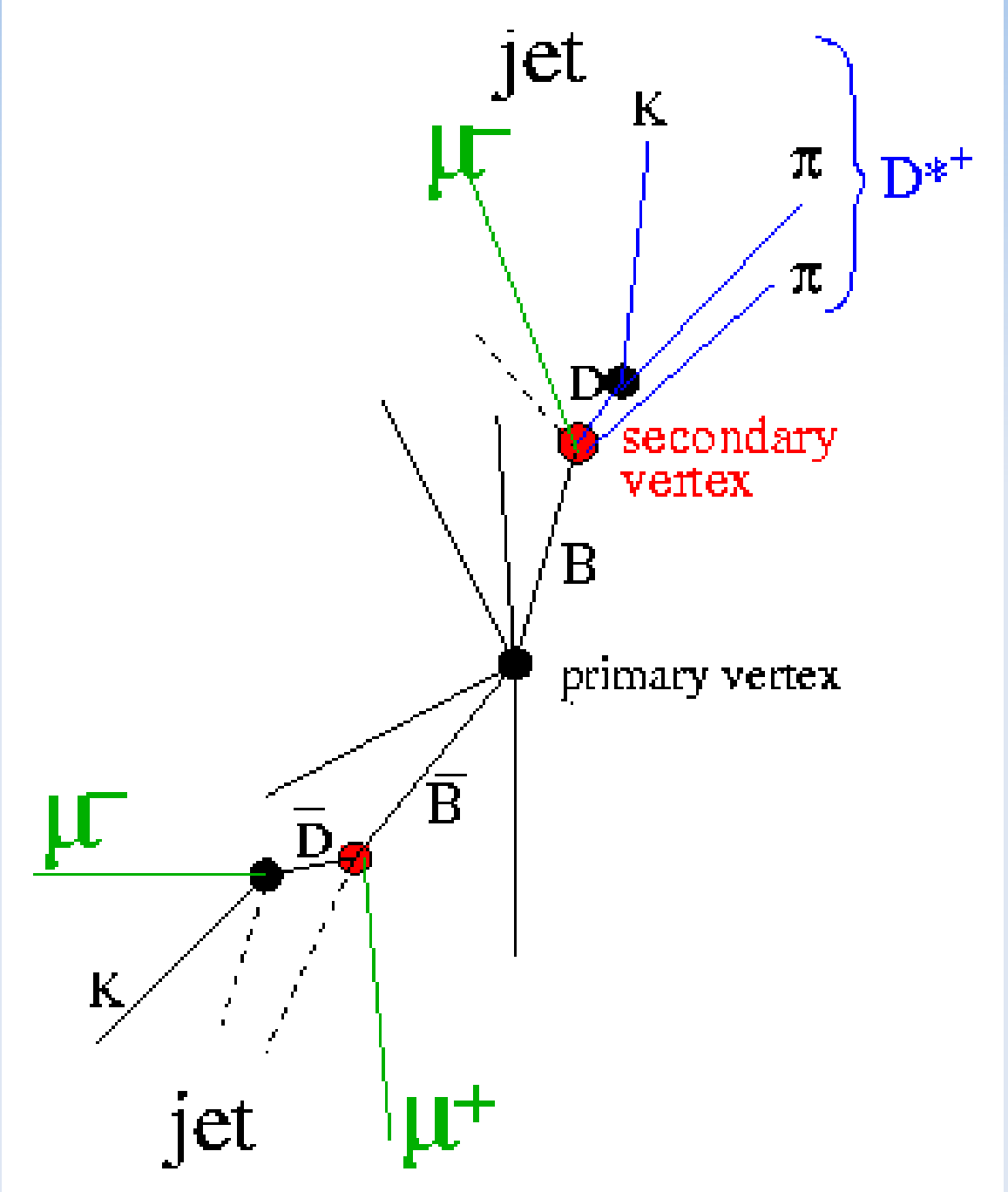




\section{b $\bar{b}$ Production}

\section{ZEUS}

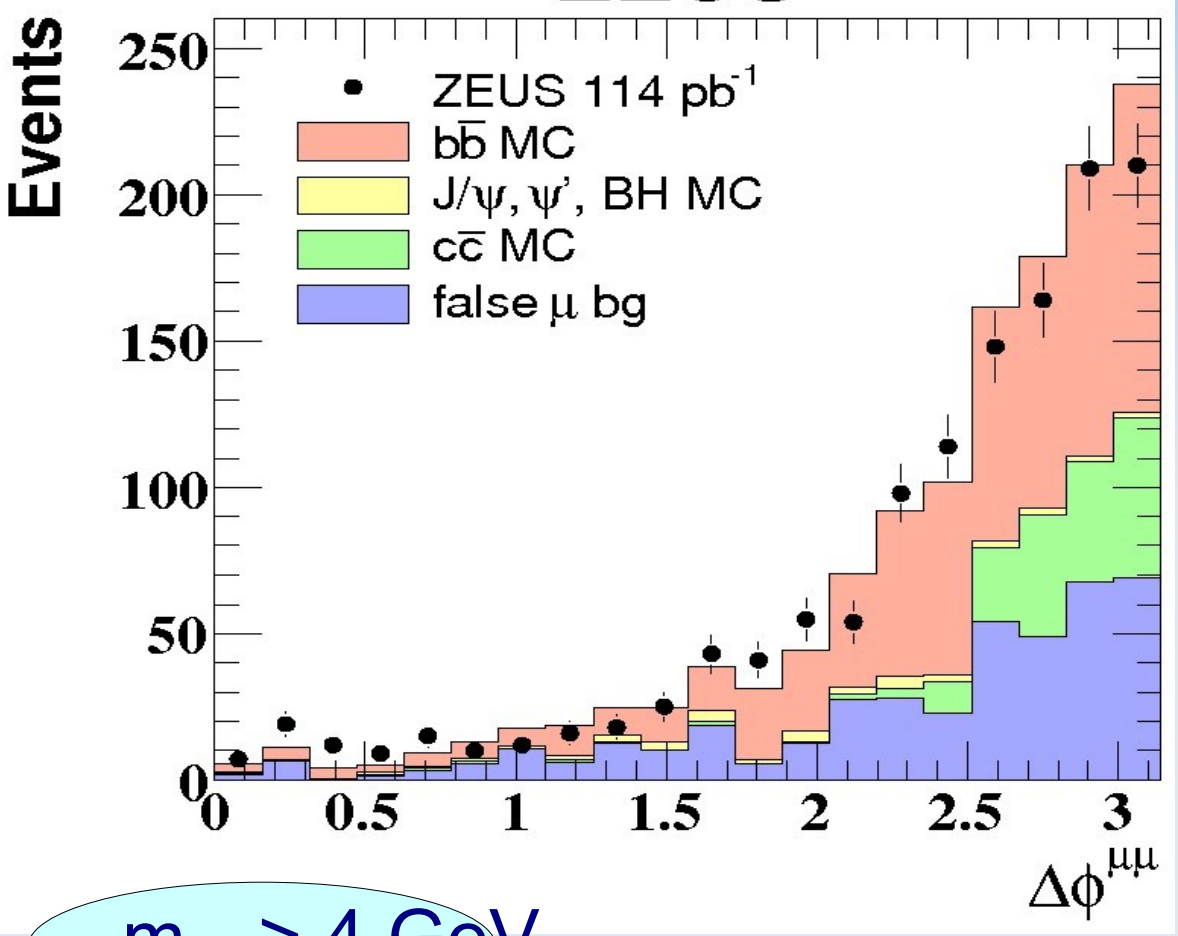

\section{ZEUS}

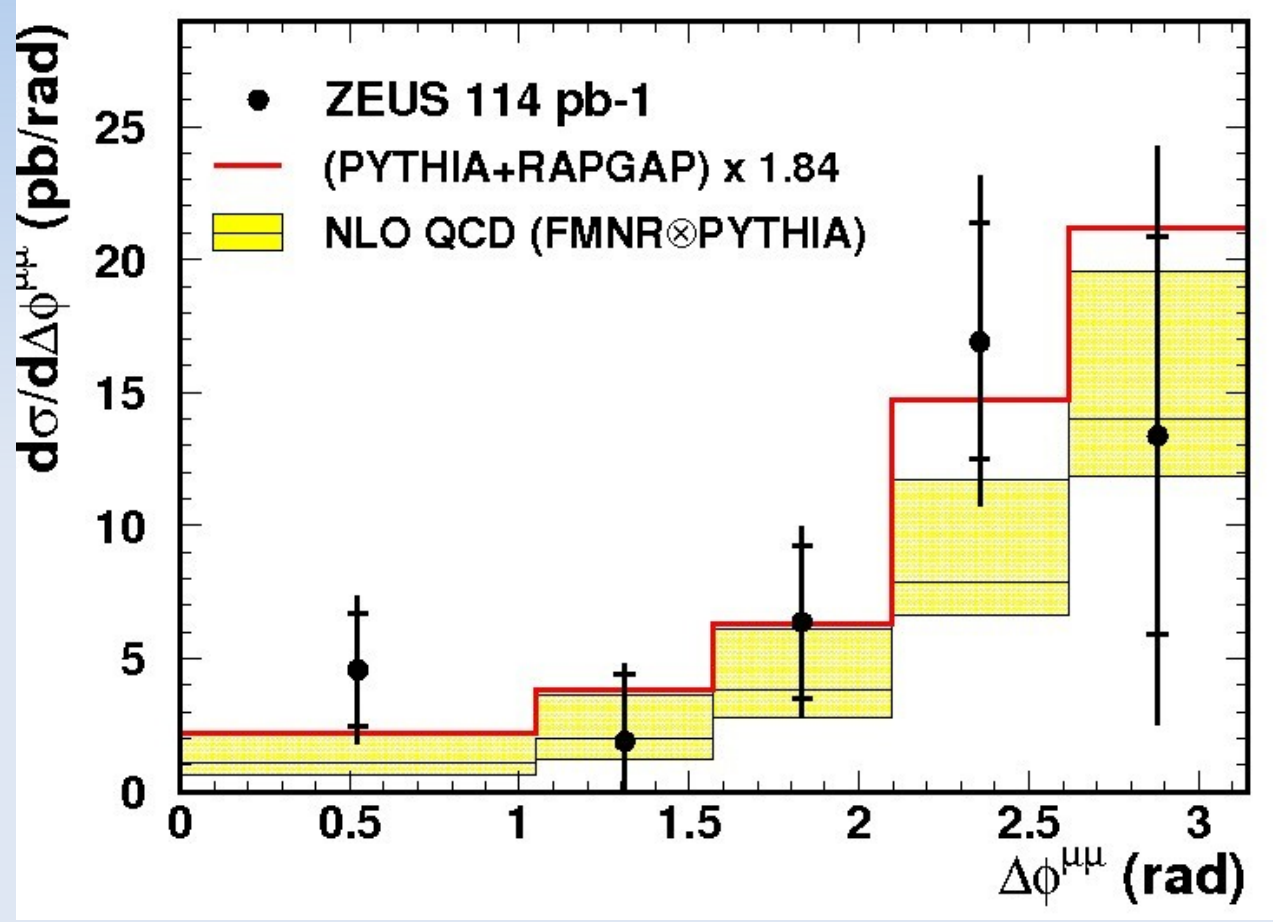

- $\Delta \varphi$ between muons from different quarks

- Correlations reasonably well described 


\section{Summary of b Photoproduction}

\section{HERA}

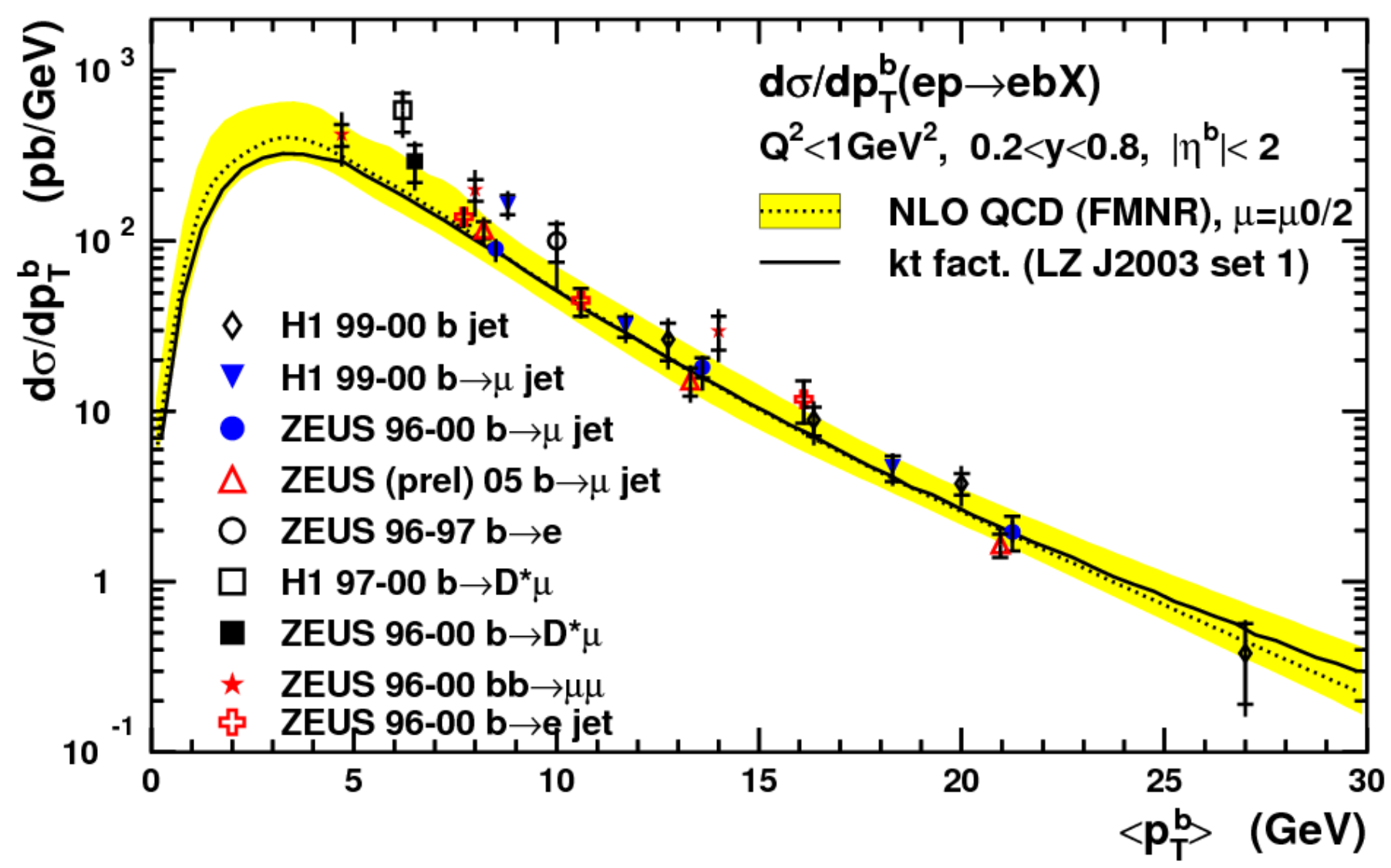

Plot actually shows ratio of measurement to FMNR prediction

\section{No sign of large excess seen in first b production measurements}




\section{b \& c in DIS}

- HERA II data

- $54 \mathrm{pb}^{-1}(2006)$

- DIS

- $\mathrm{Q}^{2}>12 \mathrm{GeV}^{2}$

- Use lifetime information

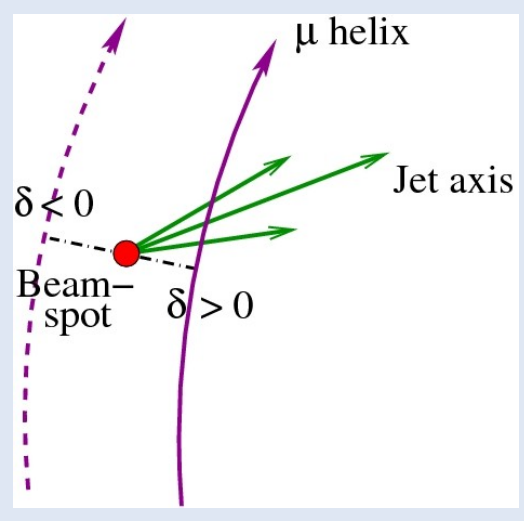

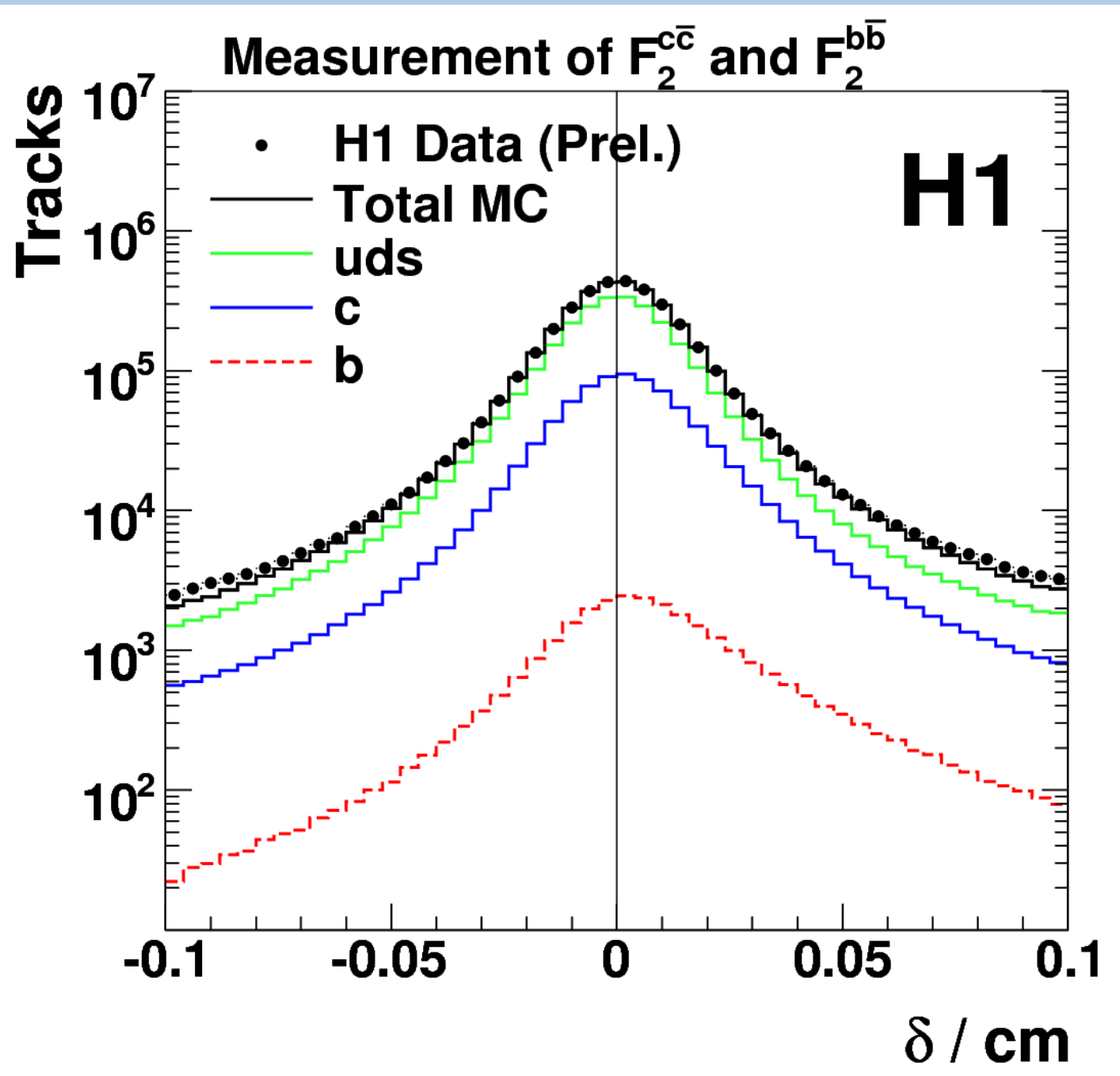

Heavy Flavour Production at HERA Ian C. Brock 


\section{b \& c in DIS}

$S_{1}$ highest

- Significance: $\delta / \sigma_{\delta}$

$\mathrm{S}_{2} 2^{\text {nd }}$ highest

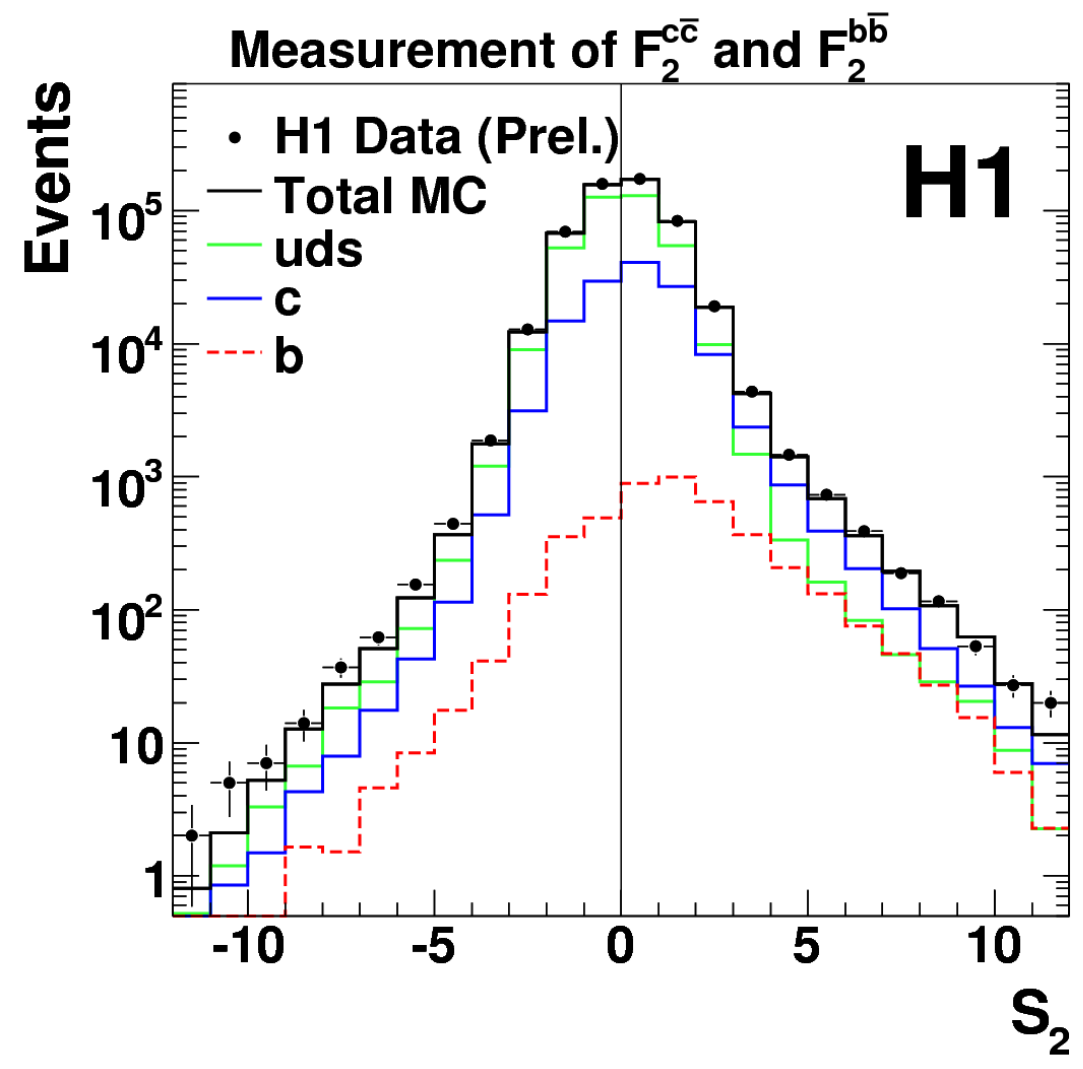

Reject events when $S_{1}$ and $S_{2}$ have opposite sign
- Subtracted significance

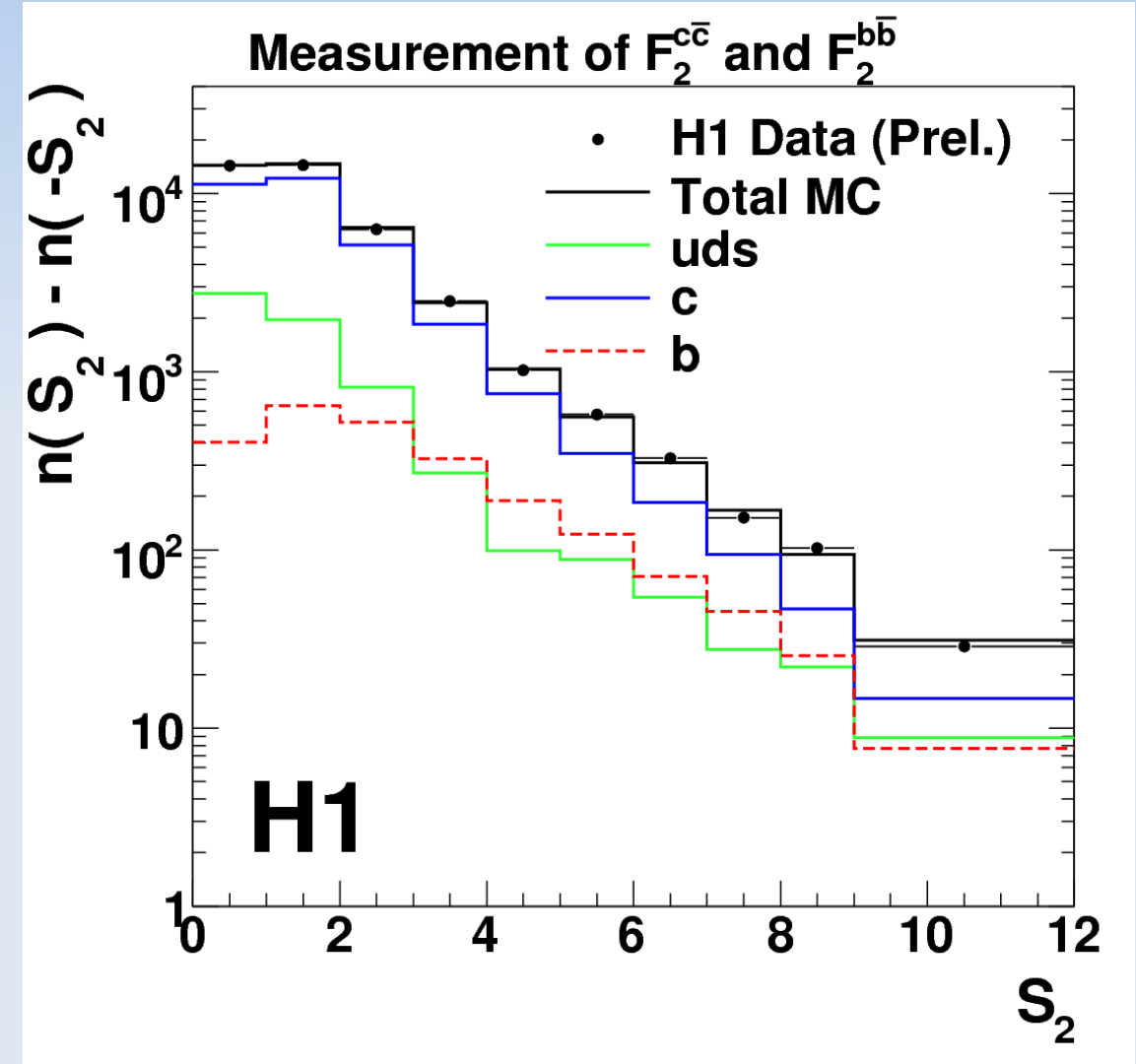

More beauty at high significance 


\section{b \& c in DIS}

- Split data into $\mathrm{Q}^{2}$ - X (Bjorken) bins

- Extract $F_{2}$ from reduced cross-sections:

$$
\tilde{\sigma}^{c \bar{c}}\left(x, Q^{2}\right)=F_{2}^{c \bar{c}}-\frac{y^{2}}{\left(1+(1-y)^{2}\right)} F_{L}^{c \bar{c}}
$$

- Combine HERA I \& HERA II measurements

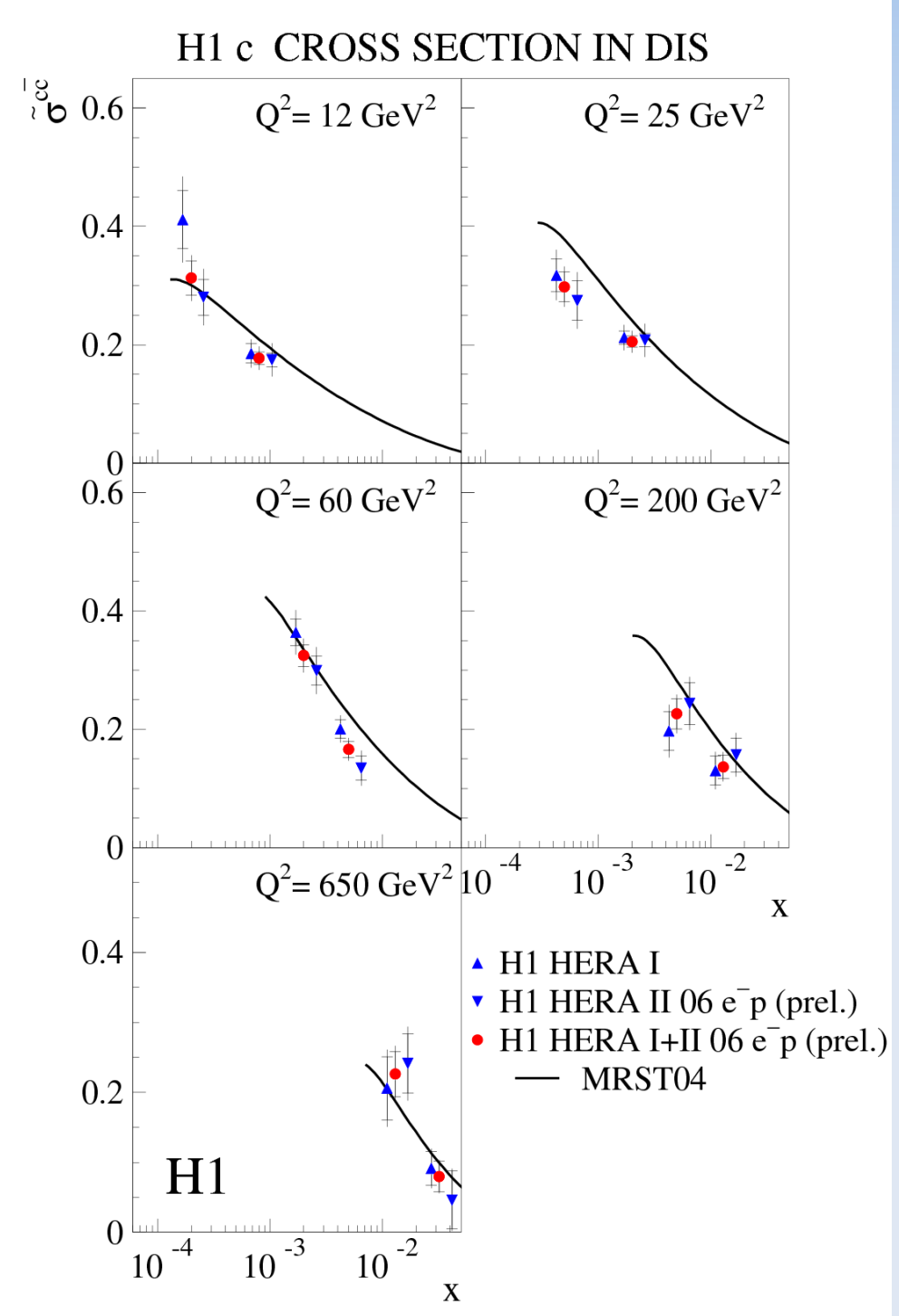




\section{b \& c in DIS}
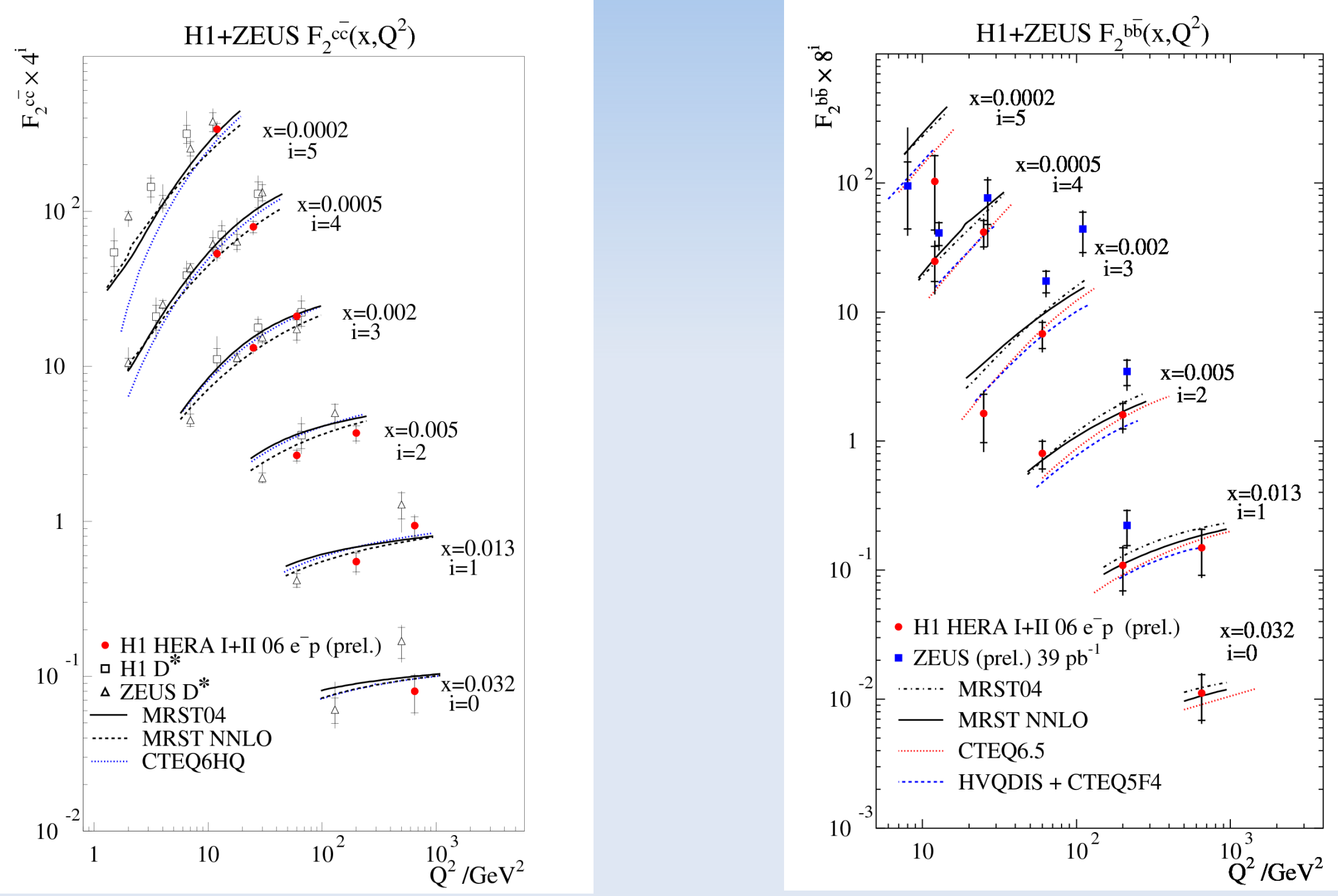

universitätbonn

Heavy Flavour Production at HERA Ian C. Brock 


\section{Conclusions}

- Small selection of HERA heavy flavour measurements presented:

- D* production

- Beauty production via semileptonic dcays to $e, \mu$

- Double $\mu$ tags

- $\mathrm{F}_{2}{ }^{\mathrm{cc}}, \mathrm{F}_{2}^{\mathrm{bb}}$

- General agreement with NLO QCD predictions

- LO Monte Carlos usually describe shape well

- Data often overshoot predictions in forward direction 


\section{Outlook}

- Several HERA I measurements still to be published

- Expand kinematic region:

- Double tags

- Semileptonic decays to electrons

- Lifetime tags

- Combine tags

- Go forward! (sensitivity to gluon PDF)

- Use improved HERA II forward tracking

- Many results with complete HERA II dataset still to come 


\section{Backup}




\section{$\mathrm{dE} / \mathrm{dx}$ in ZEUS}

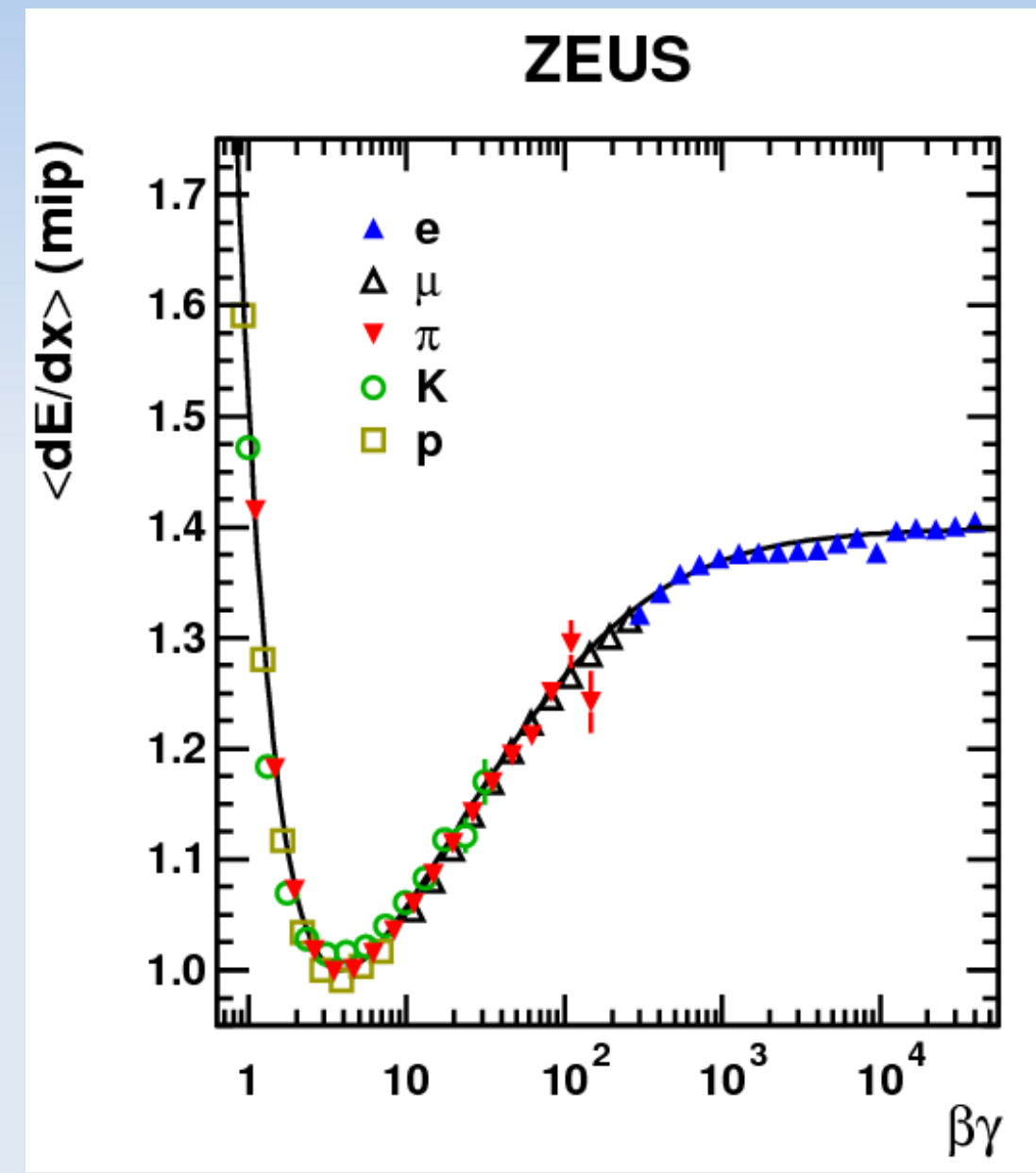

\section{ZEUS}

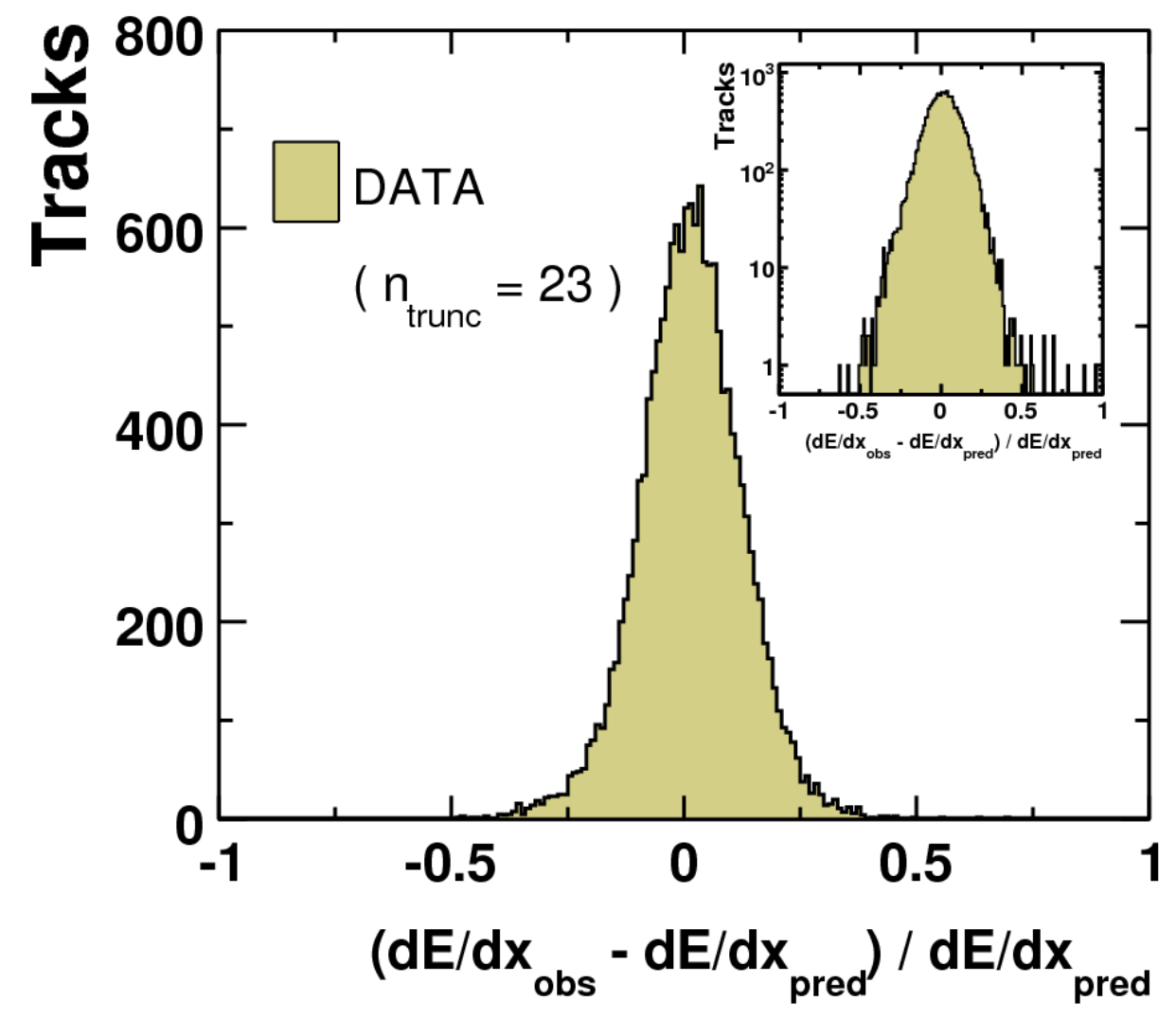

Heavy Flavour Production at HERA Ian C. Brock 


\section{b \& c in Photoproduction}

\section{- Electron identification variables}
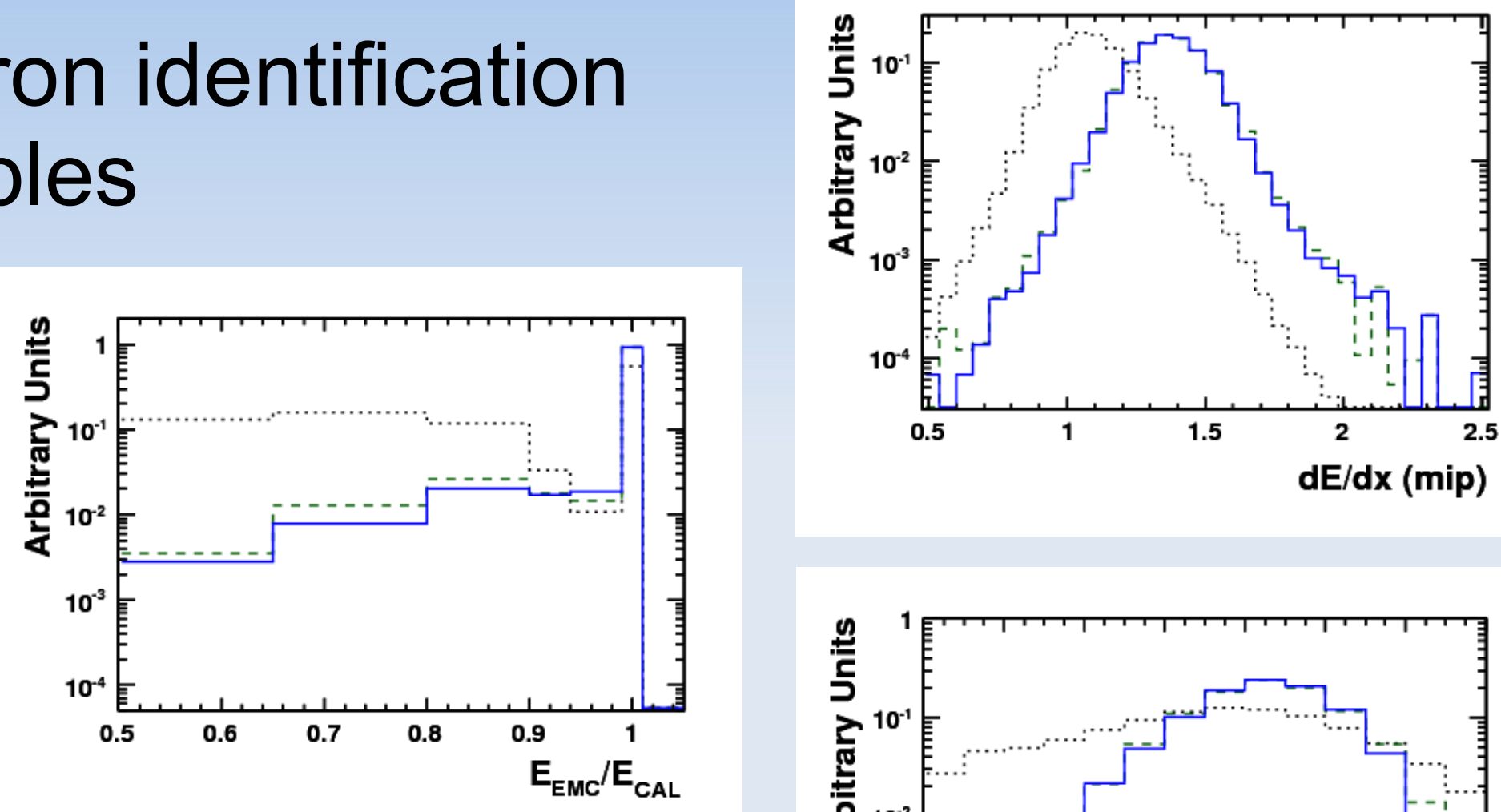

$-b \rightarrow e X$

$c \rightarrow$ e X

Bkg

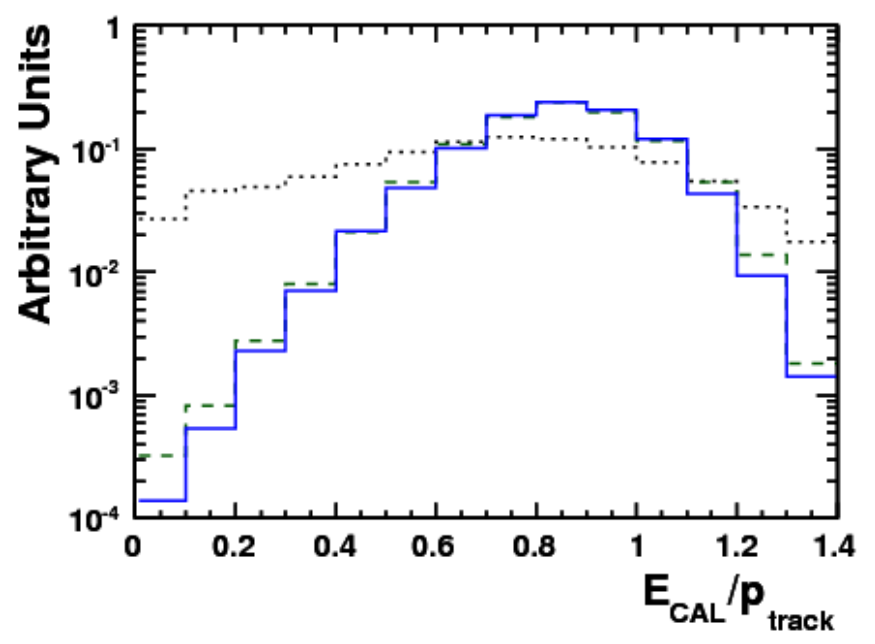




\section{b \& c in Photoproduction}

$\mathrm{E}_{\mathrm{T}}^{\text {jet1 }} \quad$ ZEUS $\eta^{\text {jet1 }}$

Beauty
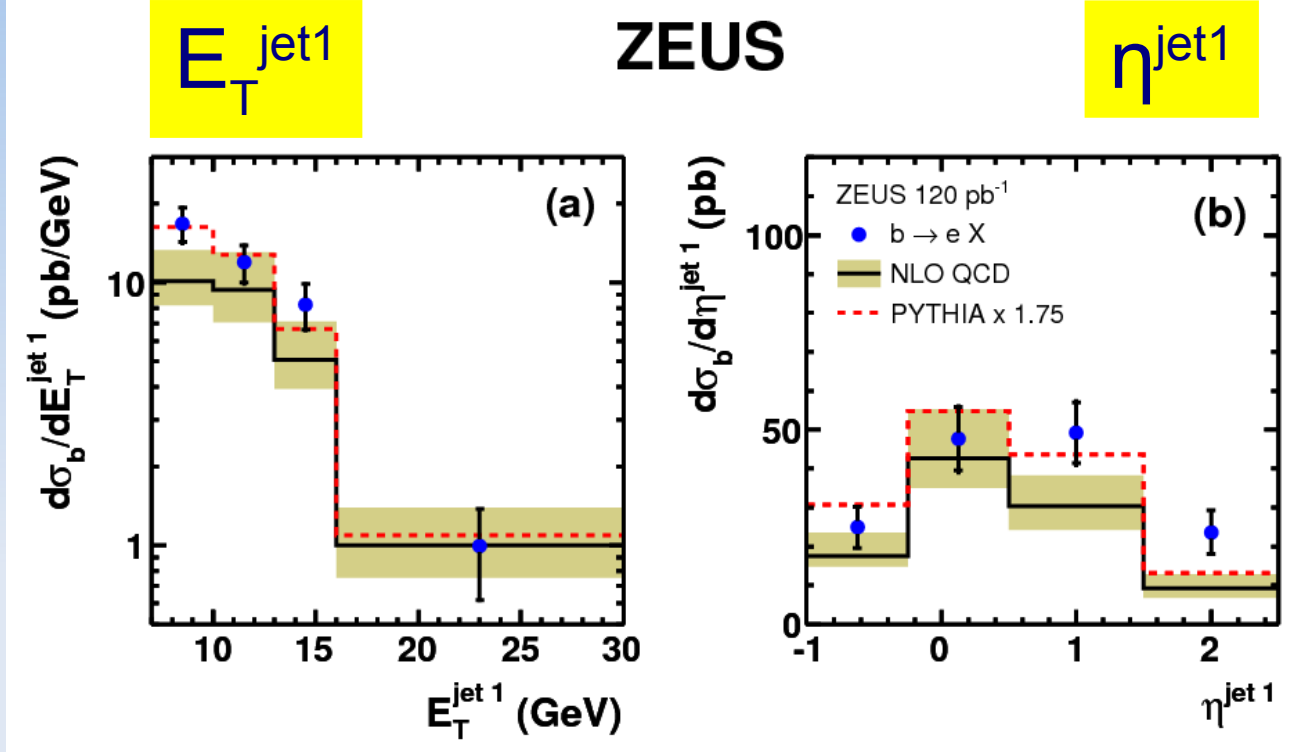

Charm

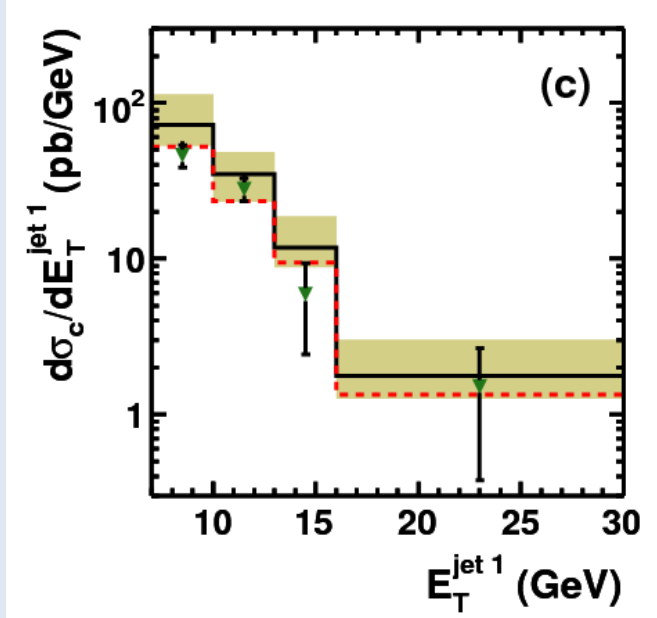

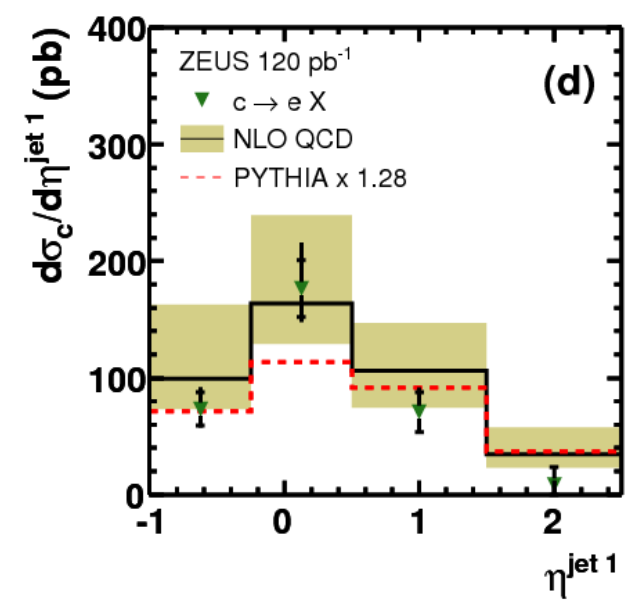

- LO Monte Carlo scale factors:

- bx 1.75

- $c \times 1.28$

- NLO absolute predictions 


\section{b $\bar{b}$ Production}

- Split into different charge combinations

- Also use $\mu \mu$ invariant mass to separate signal and background

- Most of background can be estimated from the data

\section{ZEUS}
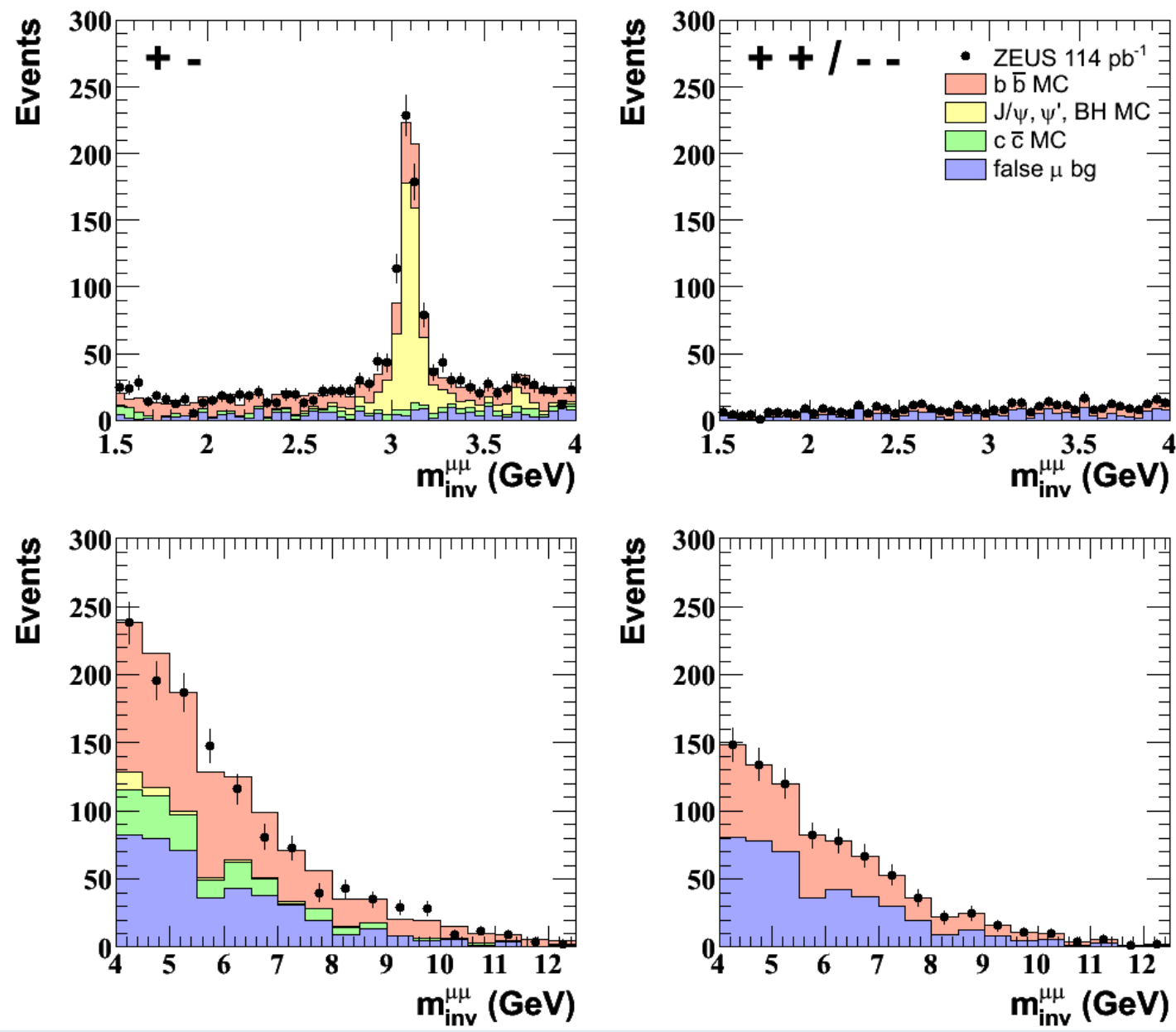


\section{b \& c in DIS}

- Significance $\left(2^{\text {nd }}\right.$ highest significance track)

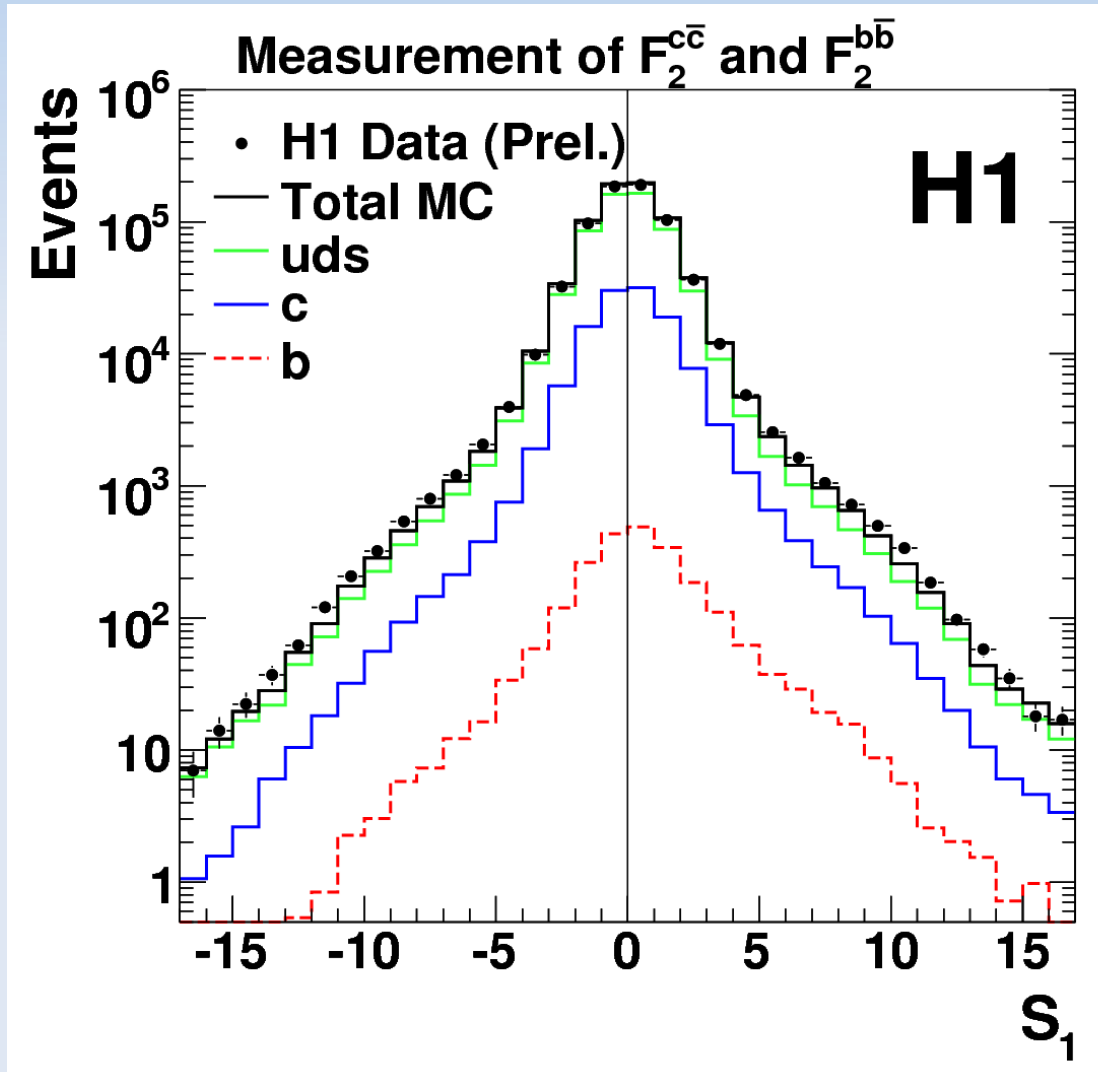

- Significance

\section{(1 track events)}

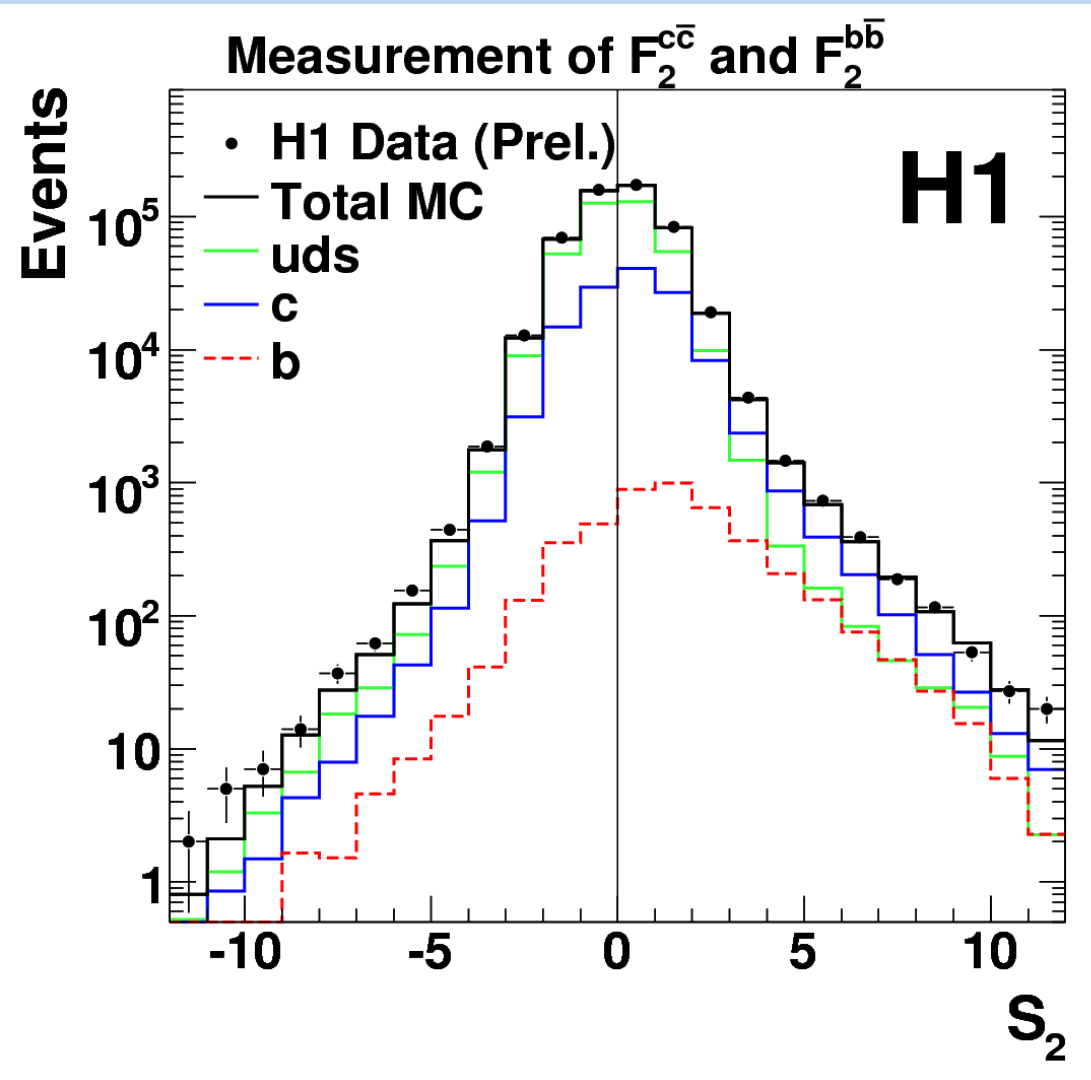

Reject events when $S_{1}$ and $S_{2}$ have opposite sign 


\section{b \& c in DIS}

\section{Subtracted significance distributions}
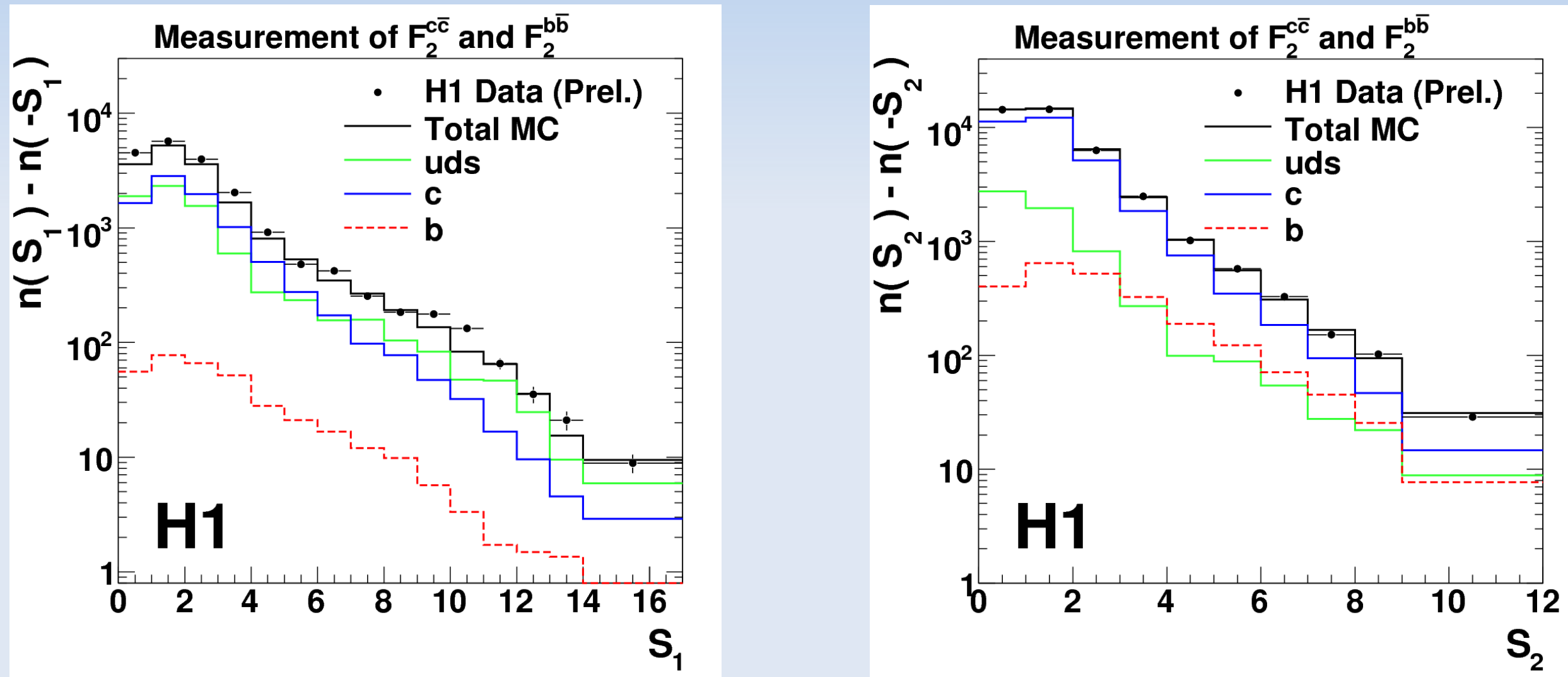


\section{Reduced Cross-Section}

$$
\begin{aligned}
& \tilde{\sigma}^{c \bar{c}}\left(x, Q^{2}\right)=\frac{d^{2} \sigma^{c \bar{c}}}{d x d Q^{2}} \frac{x Q^{4}}{2 \pi \alpha^{2}\left(1+(1-y)^{2}\right)} \\
& \tilde{\sigma}^{c \bar{c}}\left(x, Q^{2}\right)=\tilde{\sigma}\left(x, Q^{2}\right) \frac{P_{c} N_{c}^{\mathrm{MCgen}}}{P_{c} N_{c}^{\mathrm{MCgen}}+P_{b} N_{b}^{\mathrm{MCgen}}+P_{L F} N_{L F}^{\mathrm{MCgen}}} \\
& \tilde{\sigma}^{c \bar{c}}\left(x, Q^{2}\right)=F_{2}^{c \bar{c}}-\frac{y^{2}}{\left(1+(1-y)^{2}\right)} F_{L}^{c \bar{c}}
\end{aligned}
$$

